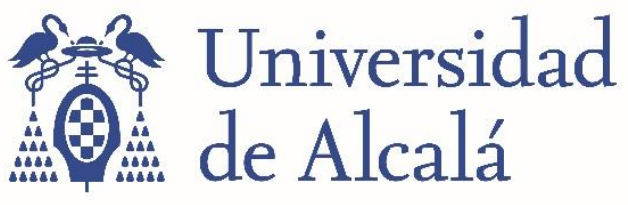

BIBLIOTECA

Document downloaded from the institutional repository of the University of Alcala: http://ebuah.uah.es/dspace/

This is a postprint version of the following published document:

Pérez Díaz, S. \& Sendra, J.R. 2013, "Behavior of the fiber and the base points of parametrizations under projections", Mathematics in Computer Science, vol. 7, no. 2, pp. 167-184.

Available at https://doi.org/10.1007/s11786-013-0139-8

(c) 2013 Springer Nature
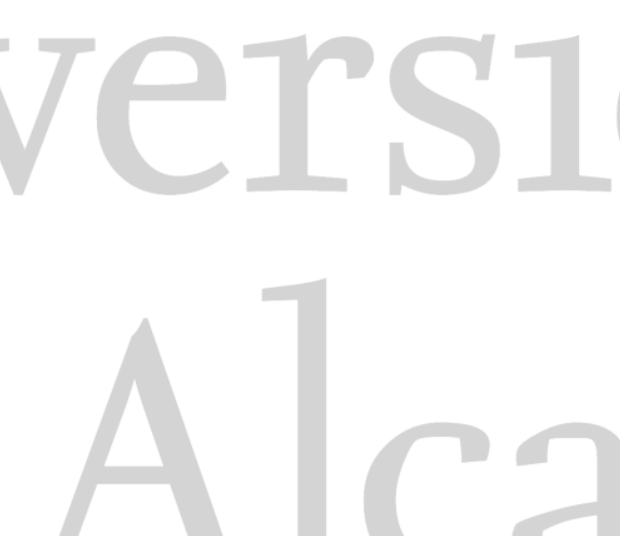

(Article begins on next page)

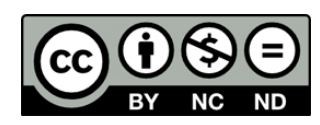

This work is licensed under a

Creative Commons Attribution-NonCommercial-NoDerivatives

4.0 International License. 


\title{
Behavior of the Fiber and the Base Points of Parametrizations under Projections
}

\author{
Sonia Pérez-Díaz and J. Rafael Sendra
}

\begin{abstract}
Given a rational parametrization $\mathcal{P}(\bar{t}), \bar{t}=\left(t_{1}, \ldots, t_{r}\right)$, of an $r$-dimensional unirational variety, we analyze the behavior of the variety of the base points of $\mathcal{P}(\bar{t})$ in connection to its generic fibre, when successively eliminating the parameters $t_{i}$. For this purpose. we introduce a sequence of generalized resultants whose primitive and content parts contain the different components of the projected variety of the base points and the fibre. In addition, when the dimension of the base points is strictly smaller than 1 (as in the well known cases of curves and surfaces), we show that the last element in the sequence of resultants is the univariate polynomial in the corresponding Gröbner basis of the ideal associated to the fibre; assuming that the ideal is in $t_{1}$-general position and radical.
\end{abstract}

Mathematics Subject Classification (2010). Primary 14Q15; Secondary 68W30, 14E05.

Keywords. Rational Parametrization, Unirational Variety, Degree of a Rational Map, Fiber of a Rational Map, Base Points, Generalized Resultants.

\section{Introduction}

We start this introduction by describing and motivating the concepts of fibre and base point as well as its relations. We consider an algebraically close field $\mathbb{K}$ of characteristic zero and a unirational variety $\mathcal{V} \subset \mathbb{K}^{n}$ of dimension $r=\operatorname{dim}(\mathcal{V})<n$. With unirational we mean that there exists a tuple of rational functions (i.e. a rational parametrization)

$$
\mathcal{P}(\bar{t})=\left(\frac{p_{1}(\bar{t})}{q(\bar{t})}, \ldots, \frac{p_{n}(\bar{t})}{q(\bar{t})}\right) \in \mathbb{K}(\bar{t})^{n}, \text { where } \bar{t}=\left(t_{1}, \ldots, t_{r}\right)
$$

(say w.l.o.g. that $\operatorname{gcd}\left(p_{1}, \ldots, p_{n}, q\right)=1$ and that none $p_{i} / q$ is constant) depending on $r$ independent parameters, $t_{1}, \ldots, t_{r}$, such that the rank of the jacobian of $\mathcal{P}(\bar{t})$ is $r$, and such that

- for almost all (i.e. for a non-empty open Zariski subset of $\mathcal{V}$ ) points $P \in \mathcal{V}$ there exists, at least one, $\bar{t}^{0} \in \mathbb{K}^{r}$ such that $P=\mathcal{P}\left(\bar{t}^{0}\right)$, and

- for all $\bar{t}^{0}$, where $\mathcal{P}(\bar{t})$ is defined, $\mathcal{P}\left(\bar{t}^{0}\right) \in \mathcal{V}$.

Associated with $\mathcal{P}(\bar{t})$ we want to define a map. Clearly it can be done as follows:

$$
\begin{aligned}
\Phi_{\mathcal{P}}: \mathbb{K}^{r} \backslash \Lambda & \rightarrow \mathcal{V} \\
\bar{t}^{0} & \mapsto \mathcal{P}\left(\bar{t}^{0}\right)
\end{aligned}
$$

This work has been partially supported by the Spanish Ministerio de Ciencia e Innovación under the project MTM200804699-C03-01 and by the Ministerio de Economía y Competitividad under the project MTM2011-25816-C02-01; both authors are members of the of the Research Group ASYNACS (Ref. CCEE2011/R34). 
where $\Lambda=\left\{\bar{t} \in \mathbb{K}^{r} \mid q(\bar{t})=0\right\}$. Note that, because of the two requirements above, $\Phi_{\mathcal{P}}$ is well defined and $\Phi_{\mathcal{P}}\left(\mathbb{K}^{r} \backslash \Lambda\right)$ is dense (in the Zariski topology) in $\mathcal{V}$. At this point, the first natural questions appear: what is the cardinality of $\Phi_{\mathcal{P}}^{-1}(P)$ for a generic point $P \in \Phi_{\mathcal{P}}\left(\mathbb{K}^{r} \backslash \Lambda\right)$ ? If this cardinality is bigger than 1 , can we replace $\mathcal{P}(\bar{t})$ by another parametrization where this cardinality is 1 ?

The cardinality of $\Phi_{\mathcal{P}}^{-1}(P)$ is the degree of $\Phi_{\mathcal{P}}$ (see e.g. [9] for a formal definition) and the set $\Phi_{\mathcal{P}}^{-1}(P)$ is called the (generic) fibre of $\Phi_{\mathcal{P}}$; we denote the fibre by $\mathfrak{F}_{\mathcal{P}}(\bar{h})$, where the generic point $P$ has been taken as $\mathcal{P}(\bar{h})$, being $\bar{h}$ a new tuple of parameters. When the degree of $\Phi_{\mathcal{P}}$ is 1 , we say that the parametrization is rational and, hence, the second question asks whether the concepts of rationality and unirationality are equivalent; question that is related to Lüroth's theorem and Castelnouvo's theorem and that we do not deal with here.

Coming back to the definition of $\Phi_{\mathcal{P}}$, one may try to get information on the parameter values in $\Lambda$. Intuitively, they might be related to the points of $\mathcal{V}$ at infinity. For a deeper analysis of this, we pass to the projective space. That is, we consider the projective closure $\mathcal{V}^{H}$ of $\mathcal{V}$. Moreover we consider the projective parametrization $\mathcal{P}^{H}\left(\bar{t}^{H}\right)$ associated with $\mathcal{P}(\bar{t})$; that is, $\bar{t}^{H}=\left(t_{0}: t_{1}: \ldots: t_{r}\right)$ and

$$
\mathcal{P}^{H}\left(\bar{t}^{H}\right)=\left(p_{1}^{H}\left(\bar{t}^{H}\right): \ldots: p_{n}^{H}\left(\bar{t}^{H}\right): q^{H}\left(\bar{t}^{H}\right)\right),
$$

where $p_{i}^{H}, q^{H}$ are the homogenization of $p_{i}, q$, respectively, multiplied by a suitable power of $t_{0}$ such that all the homogeneous polynomials $p_{i}^{H}, q^{H}$ have the same degree and $\operatorname{gcd}\left(p_{1}^{H}, \ldots, p_{n}^{H}, q^{H}\right)=1$. In this situation, we try to define a projective map, using $\mathcal{P}^{H}\left(\bar{t}^{H}\right)$, from $\mathbb{P}^{r}(\mathbb{K})$ on $\mathcal{V}^{H}$. This can be done as follows:

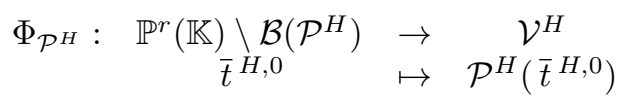

where $\mathcal{B}\left(\mathcal{P}^{H}\right)=\left\{\bar{t}^{H} \in \mathbb{P}^{r}(\mathbb{K}) \mid p_{1}^{H}\left(\bar{t}^{H}\right)=\cdots=p_{n}^{H}\left(\bar{t}^{H}\right)=q\left(\bar{t}^{H}\right)=0\right\}$. The points in $\mathcal{B}\left(\mathcal{P}^{H}\right)$ are called the (projective) base points of $\mathcal{P}^{H}\left(\bar{t}^{H}\right)$. Since, our starting object was $\mathcal{P}(\bar{t})$, we are interested in $\mathcal{B}\left(\mathcal{P}^{H}\right) \cap\left\{\bar{t}^{H} \in \mathbb{P}^{r}(\mathbb{K}) \mid t_{0} \neq 0\right\}$, that we can identify with the set $\left\{\bar{t} \in \mathbb{K}^{r} \mid p_{1}(\bar{t})=\right.$ $\left.\cdots=p_{n}(\bar{t})=q(\bar{t})=0\right\}$; in the next paragraph we will extended this definition considering this variety defined over a superfield of $\mathbb{K}$. We call these points the (affine) base points of $\mathcal{P}(\bar{t})$, and we denote the set of affine base points by $\mathfrak{B}(\mathcal{P})$. These are the points we deal with here; see e.g. [14] for further comments on projective base points.

But, how are the base points related to the generic fiber? In order to define the fibre we have considered a new tuple of parameters $\bar{h}$. Let $\mathbb{F}$ be the algebraic closure of $\mathbb{K}(\bar{h})$. Then, the generic fibre consists in those $\bar{t}^{0} \in \mathbb{F}^{r}$ such that $\mathcal{P}\left(\bar{t}^{0}\right)=\mathcal{P}(\bar{h})$. Therefore if $V_{1}$ is variety defined by the polynomials $\left\{p_{i}(\bar{t}) q(\bar{h})=q(\bar{t}) p_{i}(\bar{h})\right\}_{i=1, \ldots, r}$ and $V_{2}$ the the variety defined by $\{q(\bar{t})\}$, both over $\mathbb{F}$, then

$$
\mathfrak{F}_{\mathcal{P}}(\bar{h})=V_{1} \backslash V_{2} .
$$

On the other hand, the base points is the variety defined over $\mathbb{K}$ by $\left\{p_{1}, \ldots, p_{r}, q\right\}$. Now, let us see the $\mathfrak{B}(\mathcal{P})$ defined over $\mathbb{F}$ instead that over $\mathbb{K}$; we call it again $\mathfrak{B}(\mathcal{P})$. Then

$$
\mathfrak{F}_{\mathcal{P}}(\bar{h})=V_{1} \backslash \mathfrak{B}(\mathcal{P}) .
$$

Beside the above motivation on the definability of the rational map associated to the parametrization, why are the base points so important? The computation of the fibre, the degree of the map, the implicit equations, the singularities of $\mathcal{V}$, etc, all these questions can be translated to elimination theory problems and, consequently, approached by means of Gröbner bases or characteristic sets. Nevertheless, many authors have been and are trying to approach these associated problems by means of resultants (classical resultants, $u$-resultants, multivariate resultants, etc) appearing, for instance, in the development of the $\mu$-base, moving curves and moving surfaces theory, etc. The 
main motivation for using resultants, instead of stronger elimination techniques as Gröbner bases or characteristic sets, is not unique but essentially is based on the translation of the problem into linear algebra. This allows, for instance, the use of determinantal expressions for the implicit equations, the application of interpolation, or more generally homomorphic, techniques and provides the establishment of an easier bridge to apply numerical techniques when dealing with the corresponding approximated version of the problems.

However, most of these methods, based on resultants hit difficulties under the presence of base points (see [1], [2], [3], [4], [5], [6], [7], [13], [14]). Nevertheless, for the surface case $(r=2)$, our approaches, based on generalized resultants, to compute the implicit equation as well as the degree of the map (see [10], [11], [12]) do work even under the presence of base points. All these algorithmic methods play a crucial role in many applications, as for instance in computer aided geometric design, and therefore the theoretical understanding of the base points helps in the improvement of these potential practical applications.

Let us take a closer look at the base points of $\mathcal{P}(\bar{t})$. If $r=1, \operatorname{since} \operatorname{gcd}\left(p_{1}, \ldots, p_{n}, q\right)=1$, one has that $\mathfrak{B}(\mathcal{P})=\emptyset$. So the curve case is trivial. If $r=2, \mathfrak{B}(\mathcal{P})$ is either empty or consists in the intersection points of $(n+1)$ plane curves without common components, namely those defined by $p_{1}\left(t_{1}, t_{2}\right), \ldots, p_{n}\left(t_{1}, t_{2}\right), q\left(t_{1}, t_{2}\right)$. Therefore, if $r=2$ then either $\mathfrak{B}(\mathcal{P})=\emptyset$ or $\operatorname{dim}(\mathfrak{B}(\mathcal{P}))=0$. The situation is more complicated when $r>2$ since $\mathfrak{B}(\mathcal{P})$ is the intersection of $(n+1)$ varieties, of dimension $(r-1)$, without common components. Thus, either $\mathfrak{B}(\mathcal{P})=\emptyset$ or $\operatorname{dim}(\mathfrak{B}(\mathcal{P}))<r-2$. In Example 3, $n=5, r=3$ and $\operatorname{dim}(\mathfrak{B}(\mathcal{P}))=1$, and in Example 6, $n=5, r=3$ and $\operatorname{dim}(\mathfrak{B}(\mathcal{P}))=$ 0.

Motivated by this last fact, in this paper, we analyze the extension of the ideas in [11], [12] to the case where $r>2$. We introduce a sequence of generalized resultants associated to $\mathcal{P}(\bar{t})$ that ends in a univariate polynomial in $t_{1}$ (see Section 4 ), and such that allows us to study how the successive projections of the points in the generic fibre of $\mathcal{P}(\bar{t})$, as well as of the points in $\mathfrak{B}(\mathcal{P})$, behave (see Section 5). The fibre is zero-dimensional and hence its projections. However, the base points variety may have high dimensional components such that their projections fill the whole projection space, and hence the information of the fiber is lost. For instance, in Example 3 where $n=5$, $r=3$ and $\operatorname{dim}(\mathfrak{B}(\mathcal{P}))=1$, the second successive projection (i.e. $\left.\left(t_{1}, t_{2}, t_{3}\right) \mapsto\left(t_{1}, t_{2}\right) \mapsto t_{1}\right)$ yields to the whole line $\mathbb{K}$. To avoid this phenomenon, at each elimination step, one has to detect the hypersurface components of the projection of the base points and excluded them from the process. More precisely, say that we have eliminated $t_{i}, \ldots, t_{r}$ and we proceed to eliminate $t_{i+1}$. Then, the corresponding generalized resultant factors as its content times its primitive part. Associated to each factor we introduce a variety in the $i$-dimensional space; let us call them $\mathfrak{C}_{i}$ and $\mathfrak{M}_{i}$, respectively. Alternatively, the fibre and $\mathfrak{B}(\mathcal{P})$ are also projected onto the same space. Then the behavior of the projections of the fibre and the base points is essentially as follows:

- the fibre projects into the primitive part variety $\mathfrak{M}_{i}$ (see Theorem 5.7),

- the hypersurface components (if any) of the base points project into the content part variety $\mathfrak{C}_{i}$ (see Theorem 5.4),

- while the low dimensional components of the base points go into $\mathfrak{M}_{i}$ (see Theorem 5.7).

For analyzing the next elimination step, we control and indeed exclude the components embedded in $\mathfrak{C}_{i} \cap \mathfrak{M}_{i}$. All these problems are studied in Section 5. Finally, in Section 6, we prove that in the cases where either $\mathfrak{B}(\mathcal{P})=\emptyset$ or $\operatorname{dim}(\mathfrak{B}(\mathcal{P}))=0$, the last element in the sequence of resultants is the univariate polynomial in the corresponding Gröbner basis of the ideal associated to the fibre; assuming that the ideal is in $t_{1}$-general position and radical. This can be seen as a generalization of the results for curves and surfaces in [11] to the case $r>3$. Note that the above hypotheses, namely either $\mathfrak{B}(\mathcal{P})=\emptyset$ or $\operatorname{dim}(\mathfrak{B}(\mathcal{P}))=0$, always hold for the case of curves and surfaces. Nevertheless, 
they still can be fulfilled for unirational varieties of high dimension. When this is not the case, i.e. when $\operatorname{dim}(\mathfrak{B}(\mathcal{P}))>0$, we cannot ensure the claim in Lemma 6.1, and more precisely statement 2 (the up property), and therefore, in this case, we cannot state the connection of the generalized resultant sequence and and Gröbner bases.

We cannot finish this introduction without saying that, although from our ideas one can derive an algorithm for the computation of the degree (when $\operatorname{dim}(\mathfrak{B}(\mathcal{P}))<1$ ), this algorithm is not efficient in its current form. The inefficiency of the algorithm is essentially due to the number of new variables that the generalized resultant sequence introduces. In order to improve this situation, one might think on a probabilistic version of the algorithm where the news variables are specialized, or on a homomorphic-based technique, but we have not explored these potential approaches. On the other hand, we should emphasize that our main goal in this paper, being more theoretical than algorithmic, is to provide the first steps towards the establishment of the theoretical framework to better understanding the behavior of the base points. Let us briefly motivate this necessity. As we have said above in this introduction, the use of resultants to solve problems related to parametrizations is an active area. However, in many of these approaches, the algorithms require that the parametrization does not have base points (see [1], [2], [3], [4], [5], [6], [7]). Also, when analyzing the surjectivity of a parametrization one hits problems under the presence of base points (see [13]). Therefore, it is not only the fact of computing the base points to decide that the corresponding algorithm will not work properly. One may think on how to find (if any) a parametrization without base points or finitely many parametrizations, each of them with different based points, but covering whole variety. For this, a good understanding of the behavior can be helpful.

Parts of our proofs presented in this paper are very technical. So, we leave most of the details of the reasonings to Section 7 .

\section{Notation and Preliminary Remarks}

Throughout this paper, we will use the following notation and terminology. $\mathbb{K}$ is an algebraically closed field of characteristic zero. $\mathcal{V} \subset \mathbb{K}^{n}$ is a unirational algebraic variety, of dimension $r=$ $\operatorname{dim}(\mathcal{V})$, rationally parametrized by

$$
\mathcal{P}(\bar{t})=\left(\frac{p_{1}(\bar{t})}{q(\bar{t})}, \ldots, \frac{p_{n}(\bar{t})}{q(\bar{t})}\right) \in \mathbb{K}(\bar{t})^{n},
$$

where $\bar{t}=\left(t_{1}, \ldots, t_{r}\right)$, and such that $\operatorname{gcd}\left(p_{1}, \ldots, p_{n}, q\right)=1$.

Remark 2.1. We assume (see below) that none of the rational functions $p_{i} / q$ is constant. Furthermore, although the reasonings in this paper can be adapted for $r \in\{1,2\}$, for simplicity in the explanation, we assume that $r>2$; note that essentially case $r=1$ is treated in [15], and $r=2$ in [11].

Associated with $\mathcal{P}(\bar{t})$, we have the rational map

$$
\begin{aligned}
\Phi_{\mathcal{P}}: \mathbb{K}^{r} & \longrightarrow \mathcal{V} \\
\bar{t} & \longmapsto \mathcal{P}(\bar{t}) .
\end{aligned}
$$

Observe that $\Phi_{\mathcal{P}}\left(\mathbb{K}^{r}\right)$ is dense in $\mathcal{V}$ and that the jacobian of $\Phi_{\mathcal{P}}$ has rank $r$; being both remarks a consequence of the fact that $\mathcal{P}(\bar{t})$ is a rational parametrization. We denote by $\operatorname{deg}\left(\Phi_{\mathcal{P}}\right)$ the degree of $\Phi_{\mathcal{P}}$ (see Section 1).

Moreover, we consider the following polynomials, where the new variables $\bar{h}=\left(h_{1}, \ldots, h_{r}\right)$ and $\bar{Z}=\left(Z_{1}, \ldots, Z_{n-2}\right)$ are introduced (note that $\left.n-2>1\right)$ : 
- $G_{i}(\bar{t}, \bar{h})=p_{i}(\bar{t}) q(\bar{h})-p_{i}(\bar{h}) q(\bar{t}) \in \mathbb{K}[\bar{h}][\bar{t}]$, for $i \in\{1, \ldots, n\}$,

- $G(\bar{t}, \bar{h}, \bar{Z})=G_{2}(\bar{t}, \bar{h})+Z_{1} G_{3}(\bar{t}, \bar{h})+\cdots+Z_{n-2} G_{n}(\bar{t}, \bar{h}) \in \mathbb{K}[\bar{h}, \bar{Z}][\bar{t}]$.

Proposition 2.2. If $p_{i} / q$ is not constant (see Remark 2.1), then $G_{i}$ is not constant.

Proof. $G_{i}$ is identically zero iff $p_{i} / q$ is constant. Thus $G_{i}$ is not zero. Now, if $G_{i}(\bar{t}, \bar{h})=\lambda \in \mathbb{K}$, then $0=G_{i}(\bar{t}, \bar{t})=\lambda$ which is impossible because of our previous remark.

For a field $\mathbb{L}$ we denote by $\overline{\mathbb{L}}$ its algebraic closure. Let $\mathbb{F}=\overline{\mathbb{K}(\bar{h})}$. Moreover, if $\mathcal{G}$ is a finite set of polynomials over $\mathbb{L}$, we represent by $\mathbb{V}_{\mathbb{L}}(\mathcal{G})$, the algebraic variety defined by $\mathcal{G}$ over $\overline{\mathbb{L}}$. We introduce the algebraic sets:

- for each $i \in\{1, \ldots, n\}, \mathcal{W}_{i}^{\bar{h}}=\mathbb{V}_{\mathbb{F}}\left(G_{i}\right) \subset \mathbb{F}^{r}$.

- $\mathcal{W}_{n+1}=\mathbb{V}_{\mathbb{F}}(q) \subset \mathbb{F}^{r}$; note that $\mathcal{W}_{n+1}$ is empty if and only if $\mathcal{P}(\bar{t})$ is a polynomial parametrization.

- We denote by $\mathfrak{B}(\mathcal{P})$ the algebraic set of base points of the parametrization $\mathcal{P}(\bar{t})$, i.e. $\mathfrak{B}(\mathcal{P})$ is the variety defined by $\left\{p_{1}, \ldots, p_{n}, q\right\}$. We will see $\mathfrak{B}(\mathcal{P})$, as we have done with $\mathcal{W}_{n+1}$, embedded in $\mathbb{F}^{r}$. So $\mathfrak{B}(\mathcal{P})=\mathbb{V}_{\mathbb{F}}\left(\left\{p_{1}, \ldots, p_{n}, q\right\}\right)$. Note that then

$$
\mathfrak{B}(\mathcal{P})=\mathcal{W}_{1}^{\bar{h}} \cap \cdots \cap \mathcal{W}_{n}^{\bar{h}} \cap \mathcal{W}_{n+1} .
$$

For every $\bar{\alpha} \in \mathbb{K}^{r}$ such that $\mathcal{P}(\bar{\alpha})$ is defined, we denote by $\mathfrak{F}_{\mathcal{P}}(\bar{\alpha})$ the fibre of $\bar{\alpha}$ via $\Phi_{\mathcal{P}}$; i.e.

$$
\mathfrak{F}_{\mathcal{P}}(\bar{\alpha})=\left\{\bar{t} \in \mathbb{K}^{r} \mid \mathcal{P}(\bar{t})=\mathcal{P}(\bar{\alpha})\right\}
$$

Note that $\operatorname{deg}\left(\Phi_{\mathcal{P}}\right)$ is the cardinality of a generic fibre.

In addition, we consider a non-empty open Zariski set of $\mathbb{K}^{r}$, that we denote by $\Omega(\mathcal{P})$, such that for $\bar{\alpha} \in \Omega(\mathcal{P})$ it holds that $\operatorname{card}\left(\mathfrak{F}_{\mathcal{P}}(\bar{\alpha})\right)=\operatorname{deg}\left(\Phi_{\mathcal{P}}\right)$ (see Theorem 7.16 in [9]). Abusing of the notation, we will denote by $\mathfrak{F}_{\mathcal{P}}(\bar{h})$ the generic fibre

$$
\mathfrak{F}_{\mathcal{P}}(\bar{h})=\left\{\bar{t} \in \mathbb{F}^{r} \mid \mathcal{P}(\bar{t})=\mathcal{P}(\bar{h})\right\} .
$$

Note that $\mathfrak{F}_{\mathcal{P}}(\bar{h})=\left(\mathcal{W}_{1}^{\bar{h}} \cap \cdots \cap \mathcal{W}_{n}^{\bar{h}}\right) \backslash \mathfrak{B}(\mathcal{P})$; see e.g. Theorem 2 in [15].

Finally, if $A$ is a subset of an affine space, we will denote by $A^{*}$ its Zariski closure. Moreover, for a polynomial $g(x)$ with coefficients over a unique factorization domain, we denote by $\operatorname{LCoeff}(g, x)$ its leading coefficient w.r.t. $x$.

\section{General Assumptions and Preliminary Results}

Throughout this paper, we assume (see also Remark 2.1) the following general assumptions:

A-1 None of the rational function $p_{i} / q$ is constant and $\operatorname{gcd}\left(p_{1}, \ldots, p_{n}, q\right)=1$.

A-2 Let $\mathcal{M}$ be the subset of those polynomials in $\left\{p_{1}, \ldots, p_{n}, q\right\}$ that are not constant. We assume that the hypersurfaces in $\mathbb{K}^{r}$, defined by each of the polynomials in $\mathcal{M}$, do not pass through the point at infinity $(0: \cdots: 0: 1: 0)$, where the homogeneous variables are $\left(t_{1}, \ldots, t_{r}, w\right)$; note that this is equivalent to require that for every $g \in \mathcal{M}$ it holds that $\operatorname{deg}_{t_{r}}(g)$ is the total degree of $g$ and $\operatorname{LCoeff}\left(g, t_{r}\right) \in \mathbb{K}$.

A-3 $G_{1}$ does not divide $G_{3}$.

These assumptions imply the following proposition. 
Proposition 3.1. If assumptions A-1 and A-2 hold, the following statements hold

1. Let $G_{i}^{H}(\bar{t}, w, \bar{h})$ denote the homogenization of $G_{i}(\bar{t}, \bar{h})$ as a polynomial in $\mathbb{K}[\bar{h}][\bar{t}]$. Then $G_{i}^{H}(0, \ldots, 0,1,0, \bar{h}) \neq 0$ for $i=1, \ldots, n$.

2. For $i=1, \ldots, n, \operatorname{deg}_{t_{r}}\left(G_{i}\right)>0$ and $\operatorname{LCoeff}\left(G_{i}, t_{r}\right) \in \mathbb{K}[\bar{h}]$.

Proof. 1. If either $p_{i}$ or $q$ is constant, it follows from A-2. Otherwise, homogenizing and taking into account the total degrees of $p_{i}$ and $q$, the result follows from A-1, A-2.

2. We express $G_{i}^{H}$ as $g_{m}(\bar{t}, \bar{h})+\cdots+g_{0}(\bar{t}, \bar{h}) w^{m}$, where $g_{i}$ is homogeneous in $\bar{t}$ of degree $i$. By (i) $g_{m}$ does depend on $t_{r}$ and $\alpha=\operatorname{LCoeff}\left(G_{i}, t_{r}\right)$ only depends on $\bar{h}$.

Next, we see that the above assumptions do not imply any loss of generality.

A-1. Say w.l.o.g. that $\mathcal{P}(\bar{t})=\left(p_{1} / q, \ldots, p_{s} / q, \lambda_{s+1}, \ldots, \lambda_{n}\right)$ with $\lambda_{i} \in \mathbb{K}$ and $p_{i} / q$ non-constant, we consider the projection

$$
\pi: \mathcal{V} \rightarrow \pi(\mathcal{V})^{*} ;\left(x_{1}, \ldots, x_{n}\right) \mapsto\left(x_{1}, \ldots, x_{s}\right)
$$

and the parametrization $\mathcal{H}(\bar{t})=\pi(\mathcal{P}(\bar{t}))$ of $\pi(\mathcal{V})^{*}$. Now, since $\pi$ is birational then $\operatorname{deg}\left(\Phi_{\mathcal{P}}\right)=$ $\operatorname{deg}\left(\Phi_{\pi(\mathcal{P})}\right)$. Therefore, we can work with $\mathcal{H}(\bar{t})$ where A-1 holds.

A-2. For every $g \in \mathcal{M}$, let $\operatorname{tdeg}(g)$ denote the total degree of $g$, and let $g_{\operatorname{tdeg}(g)}(\bar{t})$ denote the homogeneous form of maximum degree of $g(\bar{t})$; i.e. of degree tdeg $(g)$. Let $\bar{\alpha}=\left(\alpha_{1}, \ldots, \alpha_{r}\right) \in$ $\mathbb{K}^{n}$, with $\alpha_{r} \neq 0$, be such that $g_{\operatorname{tdeg}(g)}(\bar{\alpha}) \neq 0$ for all $g \in \mathcal{M}$; note that, by definition, $g_{\operatorname{tdeg}(g)}$ is not identically zero, and hence $\bar{\alpha}$ always exists. We then consider the linear parameter change $\bar{t}=L\left(\bar{t}^{*}\right)$ defined by $\bar{t}=\left(t_{1}^{*}+\alpha_{1} t_{r}^{*}, \ldots, t_{r-1}^{*}+\alpha_{r-1} t_{r}^{*}, \alpha_{r} t_{r}^{*}\right)$. Now, for all $g \in \mathcal{M}$, it holds that $g\left(L\left(\bar{t}^{*}\right)\right)$ is not constant, and $\operatorname{tdeg}(g)=\operatorname{tdeg}(g(L))$. Moreover, the homogeneous form of $g(L)$ of degree $\operatorname{tdeg}(g(L))$ is of the form

$$
g_{\mathrm{tdeg}(g)}(\bar{\alpha})\left(t_{r}^{*}\right)^{\operatorname{tdeg}(g)}+h\left(t_{1}^{*}, \ldots, t_{r-1}^{*}\right) .
$$

Since $g_{\mathrm{tdeg}(g)}(\bar{\alpha}) \neq 0$, A-2 holds for $\mathcal{Q}\left(\bar{t}^{*}\right)=\mathcal{P}\left(L\left(\bar{t}^{*}\right)\right)$. Moreover, $\operatorname{deg}\left(\Phi_{\mathcal{P}}\right)=\operatorname{deg}\left(\Phi_{\mathcal{Q}}\right)$.

A-3. This assumption is not used till Section 4. Moreover, in Remark 3.4, we see that as a consequence of the other assumptions there always exists $G_{i}$ such that $G_{1}$ does not divide $G_{i}$. Hence a simple change of coordinates yields to the required condition.

Therefore, one has the following theorem.

Theorem 3.2. The above assumptions can be assumed without loss of generality.

In the following example, we illustrate the above ideas.

Example. We consider the 3-dimensional rational variety $\mathcal{V} \subset \mathbb{C}^{5}$ (so, $n=5$ and $r=3$ ) given by the parametrization

$$
\mathcal{P}(\bar{t})=\left(\frac{t_{1}}{t_{2}}, \frac{t_{1}{ }^{2} t_{3}}{t_{2}}, \frac{t_{1}{ }^{2}}{t_{2} t_{3}}, t_{2} t_{3}, \frac{t_{1}{ }^{3}}{t_{2}}\right) .
$$

[General Assumptions] Although the assumptions A-1 and A-3 are satisfied, A-2 does not hold. Therefore, we perform the linear transformation

$$
\bar{t}=L(\bar{t})=\left(t_{1}+t_{3}, t_{2}-t_{3}, t_{3}\right)
$$


to replace $\mathcal{P}(\bar{t})$ by the new parametrization

$$
\mathcal{P}(\bar{t})=\left(\frac{t_{1}+t_{3}}{t_{2}-t_{3}}, \frac{\left(t_{1}+t_{3}\right)^{2} t_{3}}{t_{2}-t_{3}}, \frac{\left(t_{1}+t_{3}\right)^{2}}{\left(t_{2}-t_{3}\right) t_{3}},\left(t_{2}-t_{3}\right) t_{3}, \frac{\left(t_{1}+t_{3}\right)^{3}}{t_{2}-t_{3}}\right) .
$$

This new parametrization fulfills all the general assumptions. The polynomials $G_{i}$ are

$$
\begin{aligned}
& G_{1}(\bar{t}, \bar{h})=\left(t_{1}+t_{3}\right) t_{3}\left(h_{2}-h_{3}\right) h_{3}-\left(h_{1}+h_{3}\right) h_{3}\left(t_{2}-t_{3}\right) t_{3} \\
& G_{2}(\bar{t}, \bar{h})=\left(t_{1}+t_{3}\right)^{2} t_{3}{ }^{2}\left(h_{2}-h_{3}\right) h_{3}-\left(h_{1}+h_{3}\right)^{2} h_{3}{ }^{2}\left(t_{2}-t_{3}\right) t_{3} \\
& G_{3}(\bar{t}, \bar{h})=\left(t_{1}+t_{3}\right)^{2}\left(h_{2}-h_{3}\right) h_{3}-\left(h_{1}+h_{3}\right)^{2}\left(t_{2}-t_{3}\right) t_{3} \\
& G_{4}(\bar{t}, \bar{h})=\left(t_{2}-t_{3}\right)^{2} t_{3}{ }^{2}\left(h_{2}-h_{3}\right) h_{3}-\left(h_{2}-h_{3}\right)^{2} h_{3}{ }^{2}\left(t_{2}-t_{3}\right) t_{3} \\
& G_{5}(\bar{t}, \bar{h})=\left(t_{1}+t_{3}\right)^{3} t_{3}\left(h_{2}-h_{3}\right) h_{3}-\left(h_{1}+h_{3}\right)^{3} h_{3}\left(t_{2}-t_{3}\right) t_{3} \\
& G(\bar{t}, \bar{h}, \bar{Z})=G_{2}+Z_{1} G_{3}+Z_{2} G_{4}+Z_{3} G_{5} .
\end{aligned}
$$

[Base Points] We analyze the base points. For this purpose, we consider the ideal $\mathrm{I}$, in $\mathcal{C}[\bar{t}]$, generated by $\left\{p_{1}, \ldots, p_{5}, q\right\}$, and we take the Gröbner basis $\mathcal{G}$ of I w.r.t. the lex order with $t_{3}>t_{2}>t_{1}$ :

$$
\mathcal{G}=\left\{t_{1}^{2}\left(t_{2}+t_{1}\right), t_{1}\left(t_{1}+t_{3}\right),-t_{1}^{2}+t_{2} t_{3},-\left(t_{1}-t_{3}\right)\left(t_{1}+t_{3}\right)\right\} .
$$

I decomposes as

$$
\mathrm{I}=\left\langle t_{1}, t_{3}\right\rangle \cap\left\langle t_{1}+t_{3}, t_{2}+t_{1}\right\rangle \cap\left\langle t_{1}, t_{2}, t_{3}\right\rangle .
$$

Thus, the base points decomposes as union of two lines, namely

$$
\mathfrak{B}(\mathcal{P})=\{(0, \lambda, 0) \mid \lambda \in \overline{\mathbb{C}(\bar{h})}\} \cup\{(-\lambda, \lambda, \lambda) \mid \lambda \in \overline{\mathbb{C}(\bar{h})}\} .
$$

In particular, we deduce that $\operatorname{dim}(\mathfrak{B}(\mathcal{P}))=1$.

[Fibre] We deal now with $\mathfrak{F}_{\mathcal{P}}(\bar{h})$. For this, we consider the ideal J, in $\mathbb{C}(\bar{h})[\rho, \bar{t}]$, generated by $\left\{G_{1}, \ldots, G_{5}, \rho q-1\right\}$, and we take the Gröbner basis $\mathcal{F}$ of J w.r.t. the lex order with $\rho>t_{3}>t_{2}>$ $t_{1}$ :

$$
\mathcal{F}=\left\{-h_{1}^{2}+t_{1}^{2},-t_{1} h_{2}+h_{1} t_{2}, h_{1} t_{3}-t_{1} h_{3},-1+\left(h_{2} h_{3}-h_{3}^{2}\right) \rho\right\} .
$$

From $\mathcal{F}$, we get that

$$
\mathfrak{F}_{\mathcal{P}}(\bar{h})=\{\bar{h},-\bar{h}\} .
$$

Therefore, $\operatorname{deg}\left(\Phi_{\mathcal{P}}\right)=2$.

In the following lemma, assuming the general assumptions, we summarize the basic properties of the varieties $\mathcal{W}_{i}^{\bar{h}}$.

\section{Lemma 3.3. It holds that}

1. $\left(\mathcal{W}_{1}^{\bar{h}} \cap \cdots \cap \mathcal{W}_{n}^{\bar{h}}\right) \backslash \mathfrak{B}(\mathcal{P}) \subset(\mathbb{F} \backslash \mathbb{K})^{r}$ and is zero-dimensional.

2. Let $\mathcal{F}$ be a finite subset of $\mathbb{K}[\bar{t}]$. If the ideal generated by $\mathcal{F}$ is zero dimensional, then $\mathbb{V}_{\mathbb{F}}(\mathcal{F}) \subset$ $\mathbb{K}^{r}$.

3. If $\operatorname{dim}(\mathfrak{B}(\mathcal{P}))=0$, then $\mathfrak{B}(\mathcal{P}) \subset \mathbb{K}^{r}$.

4. For $\bar{\alpha} \in \Omega(\mathcal{P})$, let $\mathcal{W}_{i}^{\bar{\alpha}}=\mathbb{V}_{\mathbb{K}}\left(G_{i}(\bar{t}, \bar{\alpha})\right)$. Then, $\operatorname{dim}\left(\mathcal{W}_{1}^{\bar{\alpha}} \cap \cdots \cap \mathcal{W}_{n}^{\bar{\alpha}}\right)<r-1$.

5. $\operatorname{dim}\left(\mathcal{W}_{1}^{\bar{h}} \cap \cdots \cap \mathcal{W}_{n}^{\bar{h}}\right)<r-1$.

Remark 3.4. Because of Lemma 3.3, statement $5, \operatorname{gcd}\left(G_{1}, \ldots, G_{n}\right)=1$. Therefore there exists $G_{i}$, with $i>1$, such that $G_{1}$ does not divide $G_{i}$. In particular, as imposed in assumption A-3, we can assume w.l.o.g. that $i=3$. 


\section{Generic Resultant Sequence}

In this section we introduce the notion of generic resultant sequence, and we study its first properties. For this purpose, we need some additional notation. For $i=1, \ldots, r-2$ (recall that $r>2$; see Remark 2.1), let $\bar{W}_{i}=\left(Z_{1 i}, \ldots, Z_{(n-2) i}\right)$ be a tuple of new variables. Let $\bar{W}=\left(\bar{W}_{1}, \ldots, \bar{W}_{r-2}\right)$. For $j \in\{1, \ldots, r-1\}$ we use the notation $\bar{t}^{j}=\left(t_{1}, \ldots, t_{j}\right)$. We also denote by $\operatorname{pp}_{\mathrm{var}}(M)$ and $\operatorname{cont}_{\mathrm{var}}(M)$ the primitive part and the content of the polynomial $M$ w.r.t. the set of variables var. For the following construction we observe that, by Proposition 3.1, statement 2, $G_{1}$ and $G$ do depend on $t_{r}$. Let

- $R_{r-1}=\operatorname{res}_{t_{r}}\left(G_{1}, G\right), S_{r-1}=\operatorname{pp}_{\bar{Z}}\left(R_{r-1}\right)$.

$R_{r-1} \in \mathbb{K}\left[\bar{t}^{r-1}, \bar{h}, \bar{Z}\right]$, and $S_{r-1} \in \mathbb{K}\left[\bar{t}^{r-1}, \bar{h}, \bar{Z}\right] \backslash \mathbb{K}[\bar{h}, \bar{Z}]$; see Theorem 4.3, statement

$$
\begin{aligned}
& \text { - }\left\{\begin{array}{l}
R_{r-2}=\left\{\begin{array}{l}
S_{r-1} \text { if } S_{r-1} \text { does not depend on } t_{r-1}, \text { otherwise } \\
\operatorname{res}_{t_{r-1}}\left(S_{r-1}\left(\bar{t}^{r-1}, \bar{h}, \bar{W}_{r-2}\right), S_{r-1}\left(\bar{t}^{r-1}, \bar{h}, \bar{Z}\right)\right)
\end{array}\right. \\
S_{r-2}=\left\{\begin{array}{l}
S_{r-1} \text { if } S_{r-1} \text { does not depend on } t_{r-1}, \text { otherwise } \\
\operatorname{pp}_{\bar{Z}}\left(R_{r-2}\right)
\end{array}\right.
\end{array}\right. \\
& R_{r-2}, S_{r-2} \in \mathbb{K}\left[\bar{t}^{r-2}, \bar{h}, \bar{W}_{r-2}, \bar{Z}\right] . \\
& \text { - }\left\{\begin{array}{l}
R_{r-3}= \begin{cases}S_{r-2} & \text { if } S_{r-2} \text { does not depend on } t_{r-2}, \text { otherwise } \\
\operatorname{res}_{t_{r-2}}\left(S_{r-2}\left(\bar{t}^{r-2}, \bar{h}, \bar{W}_{r-2}, \bar{W}_{r-3}\right), S_{r-2}\left(\bar{t}^{r-1}, \bar{h}, \bar{W}_{r-2}, \bar{Z}\right)\right)\end{cases} \\
S_{r-3}= \begin{cases}S_{r-2} \text { if } S_{r-2} \text { does not depend on } t_{r-2}, \text { otherwise } \\
\operatorname{pp}_{\bar{Z}}\left(R_{r-3}\right)\end{cases}
\end{array}\right. \\
& R_{r-3}, S_{r-3} \in \mathbb{K}\left[\bar{t}^{r-3}, \bar{h}, \bar{W}_{r-2}, \bar{W}_{r-3}, \bar{Z}\right] \text {. }
\end{aligned}
$$

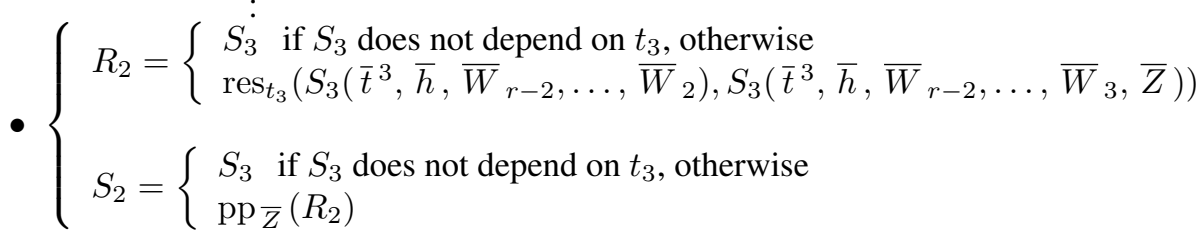

$$
\begin{aligned}
& R_{2}, S_{2} \in \mathbb{K}\left[\bar{t}^{2}, \bar{h}, \bar{W}_{r-2}, \ldots, \bar{W}_{2}, \bar{Z}\right] .
\end{aligned}
$$

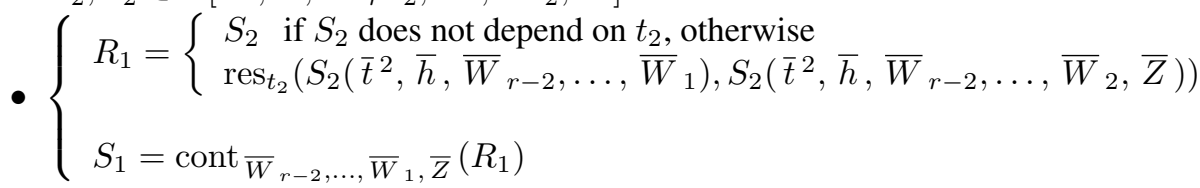

$$
\begin{aligned}
& R_{1} \in \mathbb{K}\left[t_{1}, \bar{h}, \bar{W}_{r-2}, \ldots, \bar{W}_{1}, \bar{Z}\right] \text {, and } S_{1} \in \mathbb{K}\left[t_{1}, \bar{h}\right] \text {. } \\
& \text { - } S_{0}=\operatorname{pp}_{\bar{h}}\left(S_{1}\right) \in \mathbb{K}\left[t_{1}, \bar{h}\right] \text {. }
\end{aligned}
$$

Definition 4.1. We say that $\left\{S_{0},\left(S_{1}, R_{1}\right), \ldots,\left(S_{r-1}, R_{r-1}\right)\right\}$ is the generic resultant sequence (shorten, in the following, by GRS) of $\mathcal{P}(\bar{t})$. We denote it by $\operatorname{GRS}(\mathcal{P})$.

Before establishing the basic properties on $\operatorname{GRS}(\mathcal{P})$, we state some technical lemmas on resultants and generalized resultants.

Lemma 4.2. Let $\mathbb{L}$ be a unique factorization domain. It holds that:

1. Let $M_{1}, \ldots, M_{\ell} \in \mathbb{L}[x], \ell \geq 3, M_{1}$ non-constant, and $\operatorname{gcd}\left(M_{1}, \ldots, M_{\ell}\right)=1$. $\operatorname{res}_{x}\left(M_{1}, M_{2}+\right.$ $\left.Z_{1} M_{3}+\cdots+Z_{\ell-2} M_{\ell}\right)$ does not depend on $\left\{Z_{1}, \ldots, Z_{\ell-2}\right\}$ iff $M_{1}$ divides all $M_{i}$ with $i \geq 3$. 
2. Let $\Delta$ be a tuple of variables, $M \in \mathbb{L}[\Delta][x] \backslash \mathbb{L}[x]$ without factors in $\mathbb{L}[x]$, and $N\left(\Delta^{*}, \Delta\right)=$ $\operatorname{res}_{x}\left(M\left(\Delta^{*}, x\right), M(\Delta, x)\right)$, where $\Delta^{*}$ is a tuple of new variables. $N$ depends on $\Delta$, and on $\Delta^{*}$, and has no factor depending only on $\Delta^{*}$ nor only on $\Delta$.

Theorem 4.3 (Basic Properties of GRS). Let $\operatorname{GRS}(\mathcal{P})$ be the GRS of $\mathcal{P}(\bar{t})$. It holds that:

1. $R_{i}$, for $1 \leq i \leq r-1$, depends on $\bar{Z}$; in particular it is not zero.

2. $S_{i}$, for $0 \leq i \leq r-1$, is not zero, and for $2 \leq i \leq r-1$ they depend on $\bar{Z}$, and have no factor in $\mathbb{K}[\bar{t}, \bar{h}]$.

3. $S_{r-1}$ depends on $\bar{t}^{r-1}$.

The results in the next sections use that the generic resultant sequence satisfies certain conditions on the dependencies on the variable $\bar{t}^{i}$ as well as the requirement of having constant (i.e. in $\mathbb{K}(\bar{h}, \bar{W}))$ leading coefficients; this motivates the notion of normality.

Definition 4.4. We say that $\operatorname{GRS}(\mathcal{P})$ is normal if, for $i \in\{2, \ldots, r-1\}, \operatorname{deg}_{t_{i}}\left(S_{i}\right)>0$ and LCoeff $\left(S_{i}, t_{i}\right)$ does not depend on $\bar{t}^{i-1}$.

If $\operatorname{GRS}(\mathcal{P})$ is not normal, we perform a linear transformation $\bar{t}=L\left(\bar{t}^{\prime}\right)$ such that the GRS of the transformed parametrization $\mathcal{P}\left(\bar{t}^{\prime}\right)=\mathcal{P}\left(L\left(\bar{t}^{\prime}\right)\right)$ is normal. Note that, under a linear transformation, the degree of the induced rational maps is preserved and both, base points and fibres, are under control. We have not proved that such a linear transformation exists, although empirically we have seen that for random linear transformations one yields normality.

\section{Base Points, Fibres and $\operatorname{GRS}(\mathcal{P})$}

In this section we study the connection of the base points and the fibre with the varieties defined from

$$
\operatorname{GRS}(\mathcal{P})=\left\{S_{0},\left(S_{1}, R_{1}\right), \ldots,\left(S_{r-1}, R_{r-1}\right)\right\} .
$$

To be more precise we will see how the different projections of $\mathfrak{B}(\mathcal{P})$ of $\mathfrak{F}_{\mathcal{P}}(\bar{h})$ and the varieties defined the polynomials in $\operatorname{GRS}(\mathcal{P})$ are related. For this purpose, for $r \geq \ell>i \geq 1$, we denote by $\pi_{i}$ the projection map

$$
\pi_{i}: \mathbb{F}^{\ell} \rightarrow \mathbb{F}^{i} ; \pi_{i}\left(\bar{t}^{\ell}\right)=\bar{t}^{i},
$$

and by coeffs $\mathrm{var}_{\mathbf{v a r}}(f)$ the set of coefficients of a polynomial $f$ w.r.t. the set of variables var. Moreover, we consider the fields

$$
\mathbb{F}_{j}= \begin{cases}\overline{\mathbb{K}}\left(\bar{h}, \bar{W}_{r-2}, \ldots, \bar{W}_{j}\right) & \text { if } 2 \leq j<r-1 \text { and } r>3 \\ \mathbb{F} & \text { if } j=r-1\end{cases}
$$

In addition, throughout this section we assume that $\operatorname{GRS}(\mathcal{P})$ is normal. In this situation, for $j \in$ $\{2, \ldots, r-1\}$, we consider the following varieties:

- Related to $\operatorname{GRS}(\mathcal{P})$.

$$
\mathfrak{R}_{j}=\mathbb{V}_{\mathbb{F}_{j}}\left(\operatorname{coeffs}_{\bar{Z}}\left(R_{j}\right)\right), \mathfrak{M}_{j}=\mathbb{V}_{\mathbb{F}_{j}}\left(\operatorname{coeffs}_{\bar{Z}}\left(S_{j}\right)\right), \mathfrak{C}_{j}=\mathbb{V}_{\mathbb{F}_{j}}\left(\operatorname{cont}_{\bar{Z}}\left(R_{j}\right)\right) .
$$

Note that $\mathfrak{R}_{j}=\mathfrak{C}_{j} \cup \mathfrak{M}_{j}$.

- Related to $\mathfrak{F}_{\mathcal{P}}(\bar{h})$. We decompose $\pi_{j}\left(\mathfrak{F}_{\mathcal{P}}\right)$ as $\pi_{j}\left(\mathfrak{F}_{\mathcal{P}}(\bar{h})\right)=\left(\mathfrak{F}_{\mathcal{P}}\right)_{j}^{P} \cup\left(\mathfrak{F}_{\mathcal{P}}\right)_{j}^{I}$ where

$$
\begin{cases}\left(\mathfrak{F}_{\mathcal{P}}\right)_{j}^{P}=\pi_{j}\left(\mathfrak{F}_{\mathcal{P}}(\bar{h})\right) \backslash \pi_{j}(\mathfrak{B}(\mathcal{P}))^{*} & \text { (pure part of the projection) } \\ \left(\mathfrak{F}_{\mathcal{P}}\right)_{j}^{I}=\pi_{j}\left(\mathfrak{F}_{\mathcal{P}}(\bar{h})\right) \cap \pi_{j}(\mathfrak{B}(\mathcal{P}))^{*} \quad \text { (impure part of the projection) }\end{cases}
$$


- Related to $\mathfrak{B}(\mathcal{P})$. Since $\operatorname{gcd}\left(p_{1}, \ldots, p_{n}, q\right)=1$, we have that $\operatorname{dim}(\mathfrak{B}(\mathcal{P})) \leq r-2$. Thus, $\operatorname{dim}\left(\pi_{r-1}(\mathfrak{B}(\mathcal{P}))\right) \leq r-2$. Then, we decompose $\pi_{r-1}(\mathfrak{B}(\mathcal{P}))$ as

$$
\mathbb{F}^{r-1} \supset \pi_{r-1}(\mathfrak{B}(\mathcal{P}))^{*}=\mathfrak{H}_{r-1} \cup \mathfrak{L}_{r-1}
$$

where

$\circ \mathfrak{H}_{r-1}$ is either the hypersurface contained in $\pi_{r-1}(\mathfrak{B}(\mathcal{P}))$ if $\operatorname{dim}\left(\pi_{r-1}(\mathfrak{B}(\mathcal{P}))\right)=r-2$ or, otherwise, the empty set, and

○ $\mathfrak{L}_{r-1}$ is the union of all the components of $\pi_{r-1}(\mathfrak{B}(\mathcal{P}))^{*}$ of dimension strictly smaller than $r-2$.

Additionally, we decompose $\mathfrak{M}_{r-1}$ and $\mathfrak{L}_{r-1}$ as

$$
\mathfrak{M}_{r-1}=\mathfrak{M}_{r-1}^{P} \cup \mathfrak{M}_{r-1}^{I}, \mathfrak{L}_{r-1}=\mathfrak{L}_{r-1}^{P} \cup \mathfrak{L}_{r-1}^{I}
$$

as follows:

- $\mathfrak{M}_{r-1}^{P}$ is the union of the components of $\mathfrak{M}_{r-1}$ not included in $\mathfrak{H}_{r-1}$, and $\mathfrak{M}_{r-1}^{I}$ is the union of the components of $\mathfrak{M}_{r-1}$ included in $\mathfrak{H}_{r-1}$.

- Similarly, $\mathfrak{L}_{r-1}^{P}$ is the union of the components of $\mathfrak{L}_{r-1}$ not included in $\mathfrak{H}_{r-1}$, and $\mathfrak{L}_{r-1}^{I}$ is the union of the components of $\mathfrak{L}_{r-1}$ included in $\mathfrak{H}_{r-1}$.

If $\operatorname{dim}\left(\pi_{r-2}(\mathfrak{B}(\mathcal{P}))\right)=r-2$, then $\pi_{r-2}(\mathfrak{B}(\mathcal{P}))^{*}=\mathbb{F}^{r-2}$. However, $\operatorname{dim}\left(\pi_{r-2}\left(\mathfrak{L}_{r-1}^{P}\right)\right) \leq$ $r-3$. Thus, we decompose it as

$$
\mathbb{F}^{r-2} \supset \pi_{r-2}\left(\mathfrak{L}_{r-1}^{P}\right)^{*}=\mathfrak{H}_{r-2} \cup \mathfrak{L}_{r-2}
$$

where

$\circ \mathfrak{H}_{r-2}$ is either the hypersurface contained in $\pi_{r-2}\left(\mathfrak{L}_{r-1}^{P}\right)^{*}$ if $\operatorname{dim}\left(\pi_{r-2}\left(\mathfrak{L}_{r-1}^{P}\right)\right)=r-3$ or, otherwise, the empty set, and

○ $\mathfrak{L}_{r-2}$ is the union of all the components of $\pi_{r-2}\left(\mathfrak{L}_{r-1}^{P}\right)^{*}$ of dimension strictly smaller than $r-3$.

Additionally, we decompose $\mathfrak{M}_{r-2}$ and $\mathfrak{L}_{r-2}$ as

$$
\mathfrak{M}_{r-2}=\mathfrak{M}_{r-2}^{P} \cup \mathfrak{M}_{r-2}^{I}, \quad \mathfrak{L}_{r-2}=\mathfrak{L}_{r-2}^{P} \cup \mathfrak{L}_{r-2}^{I}
$$

as follows:

- $\mathfrak{M}_{r-2}^{P}$ is the union of the components of $\mathfrak{M}_{r-2}$ not included in $\pi_{r-2}\left(\mathfrak{M}_{r-1}^{I}\right)^{*} \cup \mathfrak{H}_{r-2}$, and $\mathfrak{M}_{r-2}^{I}$ is the union of the components of $\mathfrak{M}_{r-2}$ included in $\pi_{r-2}\left(\mathfrak{M}_{r-1}^{I}\right)^{*} \cup \mathfrak{H}_{r-2}$.

- Similarly, $\mathfrak{L}_{r-2}^{P}$ is the union of the components of $\mathfrak{L}_{r-2}$ not included in $\pi_{r-2}\left(\mathfrak{M}_{r-1}^{I}\right)^{*} \cup$ $\mathfrak{H}_{r-2}$, and $\mathfrak{L}_{r-2}^{I}$ is the union of the components of $\mathfrak{L}_{r-2}$ included in $\pi_{r-2}\left(\mathfrak{M}_{r-1}^{I}\right)^{*} \cup$ $\mathfrak{H}_{r-2}$.

Repeating the argument, we decompose $\pi_{j}\left(\mathfrak{L}_{j+1}^{P}\right)$ as $\mathbb{F}^{j} \supset \pi_{j}\left(\mathfrak{L}_{j+1}^{P}\right)^{*}=\mathfrak{H}_{j} \cup \mathfrak{L}_{j}$, and we introduce $\mathfrak{M}_{j}^{P}, \mathfrak{M}_{j}^{I}, \mathfrak{L}_{j}^{P}, \mathfrak{L}_{j}^{I}$ analogously.

Remark 5.1. By definition, $\mathfrak{R}_{j}$ and $\mathfrak{M}_{j}, \mathfrak{M}_{j}^{P}, \mathfrak{M}_{j}^{I}$ are $\mathbb{F}_{j}$-definable. Furthermore, from the theorem of the closure (see [8], pp. 122) and taking into account that $\mathfrak{B}(\mathcal{P})$ is $\mathbb{K}$-definable, one deduces that $\mathfrak{H}_{j}$ and $\mathfrak{L}_{j}$ are also $\mathbb{K}$-definable. Thus, $\mathfrak{L}_{j}^{P}, \mathfrak{L}_{j}^{I}$ are also $\mathbb{K}$-definable. See also Theorem 5.8 for the $\mathbb{K}$-definability of $\mathfrak{C}_{j}$.

Remark 5.2. Observe that if $\operatorname{dim}(\mathfrak{B}(\mathcal{P}))=0$ then for $j \in\{2, \ldots, r-1\}$ it holds that $\mathfrak{H}_{j}=\mathfrak{M}_{j}^{I}=$ $\mathfrak{L}_{j}^{I}=\emptyset, \mathfrak{M}_{j}^{P}=\mathfrak{M}_{j}, \mathfrak{L}_{j}^{P}=\mathfrak{L}_{j}$ and $\operatorname{dim}\left(\mathfrak{L}_{j}\right)=0$.

Example. In this example, we illustrate the above varieties. For this purpose, we continue working with Example 3 and, hence, we use the notation introduced there. First we observe, that $\operatorname{GRS}(\mathcal{P})$ is normal. Since $r=3$ the associated varieties are: 
- For the resultant sequence: $\mathfrak{R}_{2}=\mathfrak{C}_{2} \cup \mathfrak{M}_{2}$.

- For the fibre: $\pi_{2}\left(\mathfrak{F}_{\mathcal{P}}(\bar{h})\right)=\left(\mathfrak{F}_{\mathcal{P}}\right)_{2}^{P} \cup\left(\mathfrak{F}_{\mathcal{P}}\right)_{2}^{I}$.

- For the base points: $\pi_{2}(\mathfrak{B}(\mathcal{P}))^{*}=\mathfrak{H}_{2} \cup \mathfrak{L}_{2}$ with the related decompositions

- $\mathfrak{M}_{2}=\mathfrak{M}_{2}^{P} \cup \mathfrak{M}_{2}^{I}$

- $\mathfrak{L}_{2}=\mathfrak{L}_{2}^{P} \cup \mathfrak{L}_{2}^{I}$.

[Varieties associated to $\operatorname{GRS}(\mathcal{P})$ ] The content of $R_{2}$ w.r.t. $\bar{Z}$ is $t_{1}^{2}\left(t_{2}+t_{1}\right)$. So,

$$
\mathfrak{C}_{2}=\{(0, \lambda) \mid \lambda \in \overline{\mathbb{C}(\bar{h})}\} \cup\{(-\lambda, \lambda) \mid \lambda \in \overline{\mathbb{C}(\bar{h})}\} \text {. }
$$

On the other hand, the coefficients of $S_{2}$ w.r.t. $\bar{Z}$ are

$$
\begin{aligned}
\operatorname{coeffs}_{\bar{Z}}\left(S_{2}\right)= & \left\{-\left(h_{1}+h_{3}\right)^{2}\left(h_{2}+h_{1}\right)^{2}\left(-t_{1} h_{2}+h_{1} t_{2}\right),\right. \\
& -\left(h_{1}+h_{3}\right)^{2} \Delta_{1} \Delta_{2}, \\
& -\left(h_{2}-h_{3}\right)^{2} \Delta_{1} \Delta_{2}, \\
& \left.\left(h_{1}+h_{3}\right)^{3}\left(h_{2}+h_{1}+t_{1}+t_{2}\right)\left(h_{2}+h_{1}-t_{1}-t_{2}\right) \Delta_{1}\right\}
\end{aligned}
$$

where

$$
\begin{aligned}
& \Delta_{1}=t_{1} h_{3}+h_{3} t_{2}-t_{1} h_{2}+h_{1} t_{2} \\
& \Delta_{2}=h_{2}{ }^{2} h_{3}+2 h_{1} h_{2} h_{3}+t_{1}{ }^{2} h_{2}+t_{2} h_{2} t_{1}-t_{1}{ }^{2} h_{3}-2 t_{1} h_{3} t_{2}-h_{1} t_{2} t_{1}-t_{2}{ }^{2} h_{3}+h_{1}{ }^{2} h_{3}-h_{1} t_{2}{ }^{2} .
\end{aligned}
$$

Furthermore, the Gröbner basis of $\operatorname{coeffs}_{\bar{Z}}\left(S_{2}\right)$ w.r.t. lex order with $t_{2}>t_{1}$, as ideal in $\mathbb{C}(\bar{h})\left[t_{1}, t_{2}\right]$ is

$$
\mathcal{M}=\left\{-h_{2}{ }^{2} t_{2}+t_{2}{ }^{3},-h_{1} t_{2}+t_{1} h_{2}\right\} .
$$

Therefore,

$$
\mathfrak{M}_{2}=\left\{(0,0),\left(h_{1}, h_{2}\right),\left(-h_{1},-h_{2}\right)\right\}
$$

[Fibre] In Example 3, we have seen that $\mathfrak{F}_{\mathcal{P}}(\bar{h})=\{\bar{h},-\bar{h}\}$. Thus

$$
\pi_{2}\left(\mathfrak{F}_{\mathcal{P}}(\bar{h})\right)=\left\{\left(h_{1}, h_{2}\right),\left(-h_{1},-h_{2}\right)\right\}
$$

and, taking into account $\pi_{2}(\mathfrak{B}(\mathcal{P}))^{*}$ (see below), we get that

$$
\left(\mathfrak{F}_{\mathcal{P}}\right)_{2}^{P}=\left\{\left(h_{1}, h_{2}\right),\left(-h_{1},-h_{2}\right)\right\} \text {, and }\left(\mathfrak{F}_{\mathcal{P}}\right)_{2}^{I}=\emptyset \text {. }
$$

[Base Points] In Example 3 we have seen that

$$
\mathfrak{B}(\mathcal{P})=\{(0, \lambda, 0) \mid \lambda \in \overline{\mathbb{C}(\bar{h})}\} \cup\{(-\lambda, \lambda, \lambda) \mid \lambda \in \overline{\mathbb{C}(\bar{h})}\} .
$$

Therefore, projecting the lines, one gets that

$$
\pi_{2}(\mathfrak{B}(\mathcal{P}))^{*}=\{(0, \lambda) \mid \lambda \in \overline{\mathbb{C}(\bar{h})}\} \cup\{(-\lambda, \lambda) \mid \lambda \in \overline{\mathbb{C}(\bar{h})}\} .
$$

Thus, $\pi_{2}(\mathfrak{B}(\mathcal{P}))^{*}=\mathfrak{H}_{2} \cup \mathfrak{L}_{2}$, where

$$
\mathfrak{H}_{2}=\{(0, \lambda) \mid \lambda \in \overline{\mathbb{C}(\bar{h})}\} \cup\{(-\lambda, \lambda) \mid \lambda \in \overline{\mathbb{C}(\bar{h})}\}, \text { and } \mathfrak{L}_{2}=\emptyset .
$$

Furthermore, see above, $\mathfrak{M}_{2}$ decomposes as $\mathfrak{M}_{2}=\mathfrak{M}_{2}^{P} \cup \mathfrak{M}_{2}^{I}$, where

$$
\mathfrak{M}_{2}^{P}=\left\{\left(h_{1}, h_{2}\right),\left(-h_{1},-h_{2}\right)\right\}, \text { and } \mathfrak{M}_{2}^{I}=\{(0,0)\} .
$$

Obviously $\mathfrak{L}_{2}^{P}=\mathfrak{L}_{2}^{I}=\emptyset$.

Next, we analyze the relations among the varieties we have introduced in connection to the projection of the fibre and of the base point variety. As mentioned in the introduction, we will see that essentially the behavior is as follows

- the projection of the fibre goes into the pure part $\mathfrak{M}_{i}^{P}$ of the primitive variety $\mathfrak{M}_{i}$ (see Theorem 5.7), 
- the high dimensional components of the base points project into the content variety $\mathfrak{C}_{i}$ (see Theorem 5.4),

- while the low dimensional components of the base points go into $\mathfrak{M}_{i}^{P}$ (see Theorem 5.7).

We know that

$$
\mathcal{W}_{1}^{\bar{h}} \cap \cdots \cap \mathcal{W}_{n}^{\bar{h}}=\mathfrak{F}_{\mathcal{P}}(\bar{h}) \cup \mathfrak{B}(\mathcal{P}) .
$$

We start with the next lemma where a similar decomposition holds for $\mathfrak{M}_{j}^{P}$. This first lemma, indeed, establishes that all points in the pure part of the primitives varieties are either projections of base points or of fibre points. This will be used in the next theorems to first state that the content varieties are essentially defined by the projection of the sufficiently high dimensional components of the base points (see Theorem 5.4), and to afterwards provide a clearer decomposition of the pure part of the primitive part variety (see Theorem 5.7).

Lemma 5.3. For $j \in\{2, \ldots, r-1\}, \mathfrak{M}_{j}^{P}=\left(\mathfrak{M}_{j}^{P} \cap \pi_{j}\left(\mathfrak{F}_{\mathcal{P}}(\bar{h})\right)\right) \cup\left(\mathfrak{M}_{j}^{P} \cap \mathfrak{L}_{j}^{P}\right)$.

As consequence of this lemma, we get the following theorem. We recall that the varieties introduced above are defined for $j \in\{2, \ldots, r-1\}$, where we have assumed that $r \geq 3$.

\section{Theorem 5.4 (Decomposition of $\mathfrak{C}_{j}$ ). It holds that:}

1. If $\operatorname{dim}(\mathfrak{B}(\mathcal{P}))<r-2$ then $\mathfrak{C}_{r-1}=\emptyset$.

2. If $r>3$ and $\operatorname{dim}\left(\pi_{j}\left(\left(\mathfrak{M}_{j+1}^{P} \cap \mathfrak{L}_{j+1}^{P}\right) \cup \mathfrak{M}_{j+1}^{I}\right)\right)<j-1$ for $j \in\{2, \ldots, r-2\}$, then $\mathfrak{C}_{j}=\emptyset$.

3. If $\operatorname{dim}\left(\pi_{r-1}(\mathfrak{B}(\mathcal{P}))^{*}\right)=r-2$, then $\mathfrak{C}_{r-1}=\mathfrak{H}_{r-1}$.

4. If $r>3$ and $\operatorname{dim}\left(\pi_{j}\left(\left(\mathfrak{M}_{j+1}^{P} \cap \mathfrak{L}_{j+1}^{P}\right) \cup \mathfrak{M}_{j+1}^{I}\right)\right)=j-1$ for $j \in\{2, \ldots, r-2\}$, then $\mathfrak{C}_{j}$ is the hypersurface included in $\pi_{j}\left(\left(\mathfrak{M}_{j+1}^{P} \cap \mathfrak{L}_{j+1}^{P}\right) \cup \mathfrak{M}_{j+1}^{I}\right)^{*}$.

Remark 5.5. In Example 3, we have seen that $\operatorname{dim}(\mathfrak{B}(\mathcal{P}))=1=r-2$, and in Example 5 we have seen that $\mathfrak{C}_{2}=\mathfrak{H}_{2}$; compare to Theorem 5.4, statement 3 .

Corollary 5.6. If $\operatorname{dim}(\mathfrak{B}(\mathcal{P}))=0$, for $j \in\{2, \ldots, r-1\}, \mathfrak{C}_{j}=\emptyset$.

Proof. By Theorem 5.4, statement 1, since $r>2$ (see Remark 2.1), $\mathfrak{C}_{r-1}=\emptyset$. For $j \in\{2, \ldots, r-$ $2\}$, the result follows from Theorem 5.4, statement 2, taking into account that $\pi_{j}\left(\left(\mathfrak{M}_{j+1}^{P} \cap \mathfrak{L}_{j+1}^{P}\right) \cup\right.$ $\left.\mathfrak{M}_{j+1}^{I}\right)=\pi_{j}\left(\mathfrak{M}_{j+1} \cap \mathfrak{L}_{j+1}\right) \subset \pi_{j}\left(\mathfrak{L}_{j+1}\right)$ (see Remark 5.2), from where $\operatorname{dim}\left(\pi_{j}\left(\left(\mathfrak{M}_{j+1}^{P} \cap \mathfrak{L}_{j+1}^{P}\right) \cup\right.\right.$ $\left.\left.\mathfrak{M}_{j+1}^{I}\right)\right) \leq \operatorname{dim}\left(\pi_{j}\left(\mathfrak{L}_{j+1}\right)\right)=0<j-1$.

Theorem 5.7. [Decomposition of $\mathfrak{R}_{j}, \mathfrak{M}_{j}$ ] It holds that:

1. $\mathfrak{R}_{r-1}=\pi_{r-1}(\mathfrak{B}(\mathcal{P}))^{*} \cup\left(\mathfrak{F}_{\mathcal{P}}\right)_{r-1}^{P}$.

2. If $r>3$ and $j \in\{2, \ldots, r-2\}, \mathfrak{R}_{j}=\pi_{j}\left(\mathfrak{M}_{j+1}\right)^{*}$.

3. For $j \in\{2, \ldots, r-1\}, \mathfrak{M}_{j}^{P}=\left(\mathfrak{F}_{\mathcal{P}}\right)_{j}^{P} \cup \mathfrak{L}_{j}^{P} \cup \mathfrak{Q}_{j}$, where $\mathfrak{Q}_{j} \subset\left(\mathfrak{F}_{\mathcal{P}}\right)_{j}^{I}$.

Example. We illustrate the theorem by means of Examples 3 and 5. Let $\mathcal{L}_{1}=\{(0, \lambda) \mid \lambda \in \overline{\mathbb{C}(\bar{h})}\}$ and $\mathcal{L}_{2}=\{(-\lambda, \lambda) \mid \lambda \in \overline{\mathbb{C}(\bar{h})}\}$. Then (compare to Theorem 5.7, statement 1),

$$
\begin{aligned}
& \mathfrak{R}_{2}=\mathfrak{C}_{2} \cup \mathfrak{M}_{2}=\mathcal{L}_{1} \cup \mathcal{L}_{2} \cup\left\{(0,0),\left(h_{1}, h_{2}\right),\left(-h_{1},-h_{2}\right)\right\}= \\
& =\mathcal{L}_{1} \cup \mathcal{L}_{2} \cup\left\{\left(h_{1}, h_{2}\right),\left(-h_{1},-h_{2}\right)\right\}=\pi_{2}(\mathfrak{B}(\mathcal{P}))^{*} \cup\left(\mathfrak{F}_{\mathcal{P}}\right)_{2}^{P}
\end{aligned}
$$

Moreover (compare to Theorem 5.7, statement 3), $\mathfrak{M}_{2}=\left(\mathfrak{F}_{\mathcal{P}}\right)_{2}^{P} \cup \mathfrak{L}_{2} \cup \mathfrak{Q}_{2}$, with $\mathfrak{Q}_{2}=\emptyset$. 
Theorem 5.8. [ $\mathbb{K}$-definability of $\mathfrak{C}_{j}$ ]

1. $\mathfrak{C}_{r-1}$ is $\mathbb{K}$-definable.

2. If $r>3$ and $\operatorname{dim}\left(\pi_{j}\left(\mathfrak{M}_{j+1}^{I}\right)^{*}\right)<j-1$ for $j \in\{2, \ldots, r-2\}$, then $\mathfrak{C}_{j}$ is $\mathbb{K}$-definable.

Proof. By Theorem 5.4, we only need to prove the theorem if $j<r-1$ and $\operatorname{dim}\left(\pi_{j}\left(\left(\mathfrak{M}_{j+1}^{P} \cap \mathfrak{L}_{j+1}^{P}\right) \cup\right.\right.$ $\left.\mathfrak{M}_{j+1}^{I}\right)=j-1$. By Theorem 5.4, $\mathfrak{C}_{j}$ is the hypersurface included in $\pi_{j}\left(\left(\mathfrak{M}_{j+1}^{P} \cap \mathfrak{L}_{j+1}^{P}\right) \cup \mathfrak{M}_{j+1}^{I}\right)^{*}$. Thus, reasoning as in the proof of Theorem 5.7, $\mathfrak{C}_{j}$ is the hypersurface included in $\Delta=\mathfrak{H}_{j} \cup$ $\pi_{j}\left(\mathfrak{M}_{j+1}^{I}\right)^{*}$. Thus, $\mathfrak{C}_{j} \subset \mathfrak{H}_{j}$. Now the theorem follows from Remark 5.1.

\section{Connection of $\operatorname{GRS}(\mathcal{P})$ to Gröbner Bases}

In the previous section we have analyzed some varieties of the $\operatorname{GRS}(\mathcal{P})$ in connection to the base points and a generic fibre. In this section, we study the connection to Gröbner bases. We assume that

$$
\operatorname{GRS}(\mathcal{P})=\left\{S_{0},\left(S_{1}, R_{1}\right), \ldots,\left(S_{r-1}, R_{r-1}\right)\right\}
$$

is normal. In addition, in the sequel, we assume that either $\mathfrak{B}(\mathcal{P})=\emptyset$ or $\operatorname{dim}(\mathfrak{B}(\mathcal{P}))=0$. We start with the following lemma.

Lemma 6.1. [Up and down property] It holds that:

1. If $P \in \mathfrak{F}_{\mathcal{P}}(\bar{h})$, then $\pi_{1}(P)$ is a root of $S_{0}\left(t_{1}\right)$.

2. If $\alpha$ is a root of $S_{0}\left(t_{1}\right)$, then there exists $P \in \mathfrak{F}_{\mathcal{P}}(\bar{h})$ such that $\pi_{1}(P)=\alpha$.

From Lemma 6.1, one directly gets the following theorem.

Theorem 6.2. $\pi_{1}\left(\mathfrak{F}_{\mathcal{P}}(\bar{h})\right)=\mathbb{V}_{\mathbb{F}}\left(S_{0}\right)$.

From this result we get the following corollaries.

Corollary 6.3. Let $\mathrm{I}$ be the ideal, in $\mathbb{K}(\bar{h})[\rho, \bar{t}]$, generated by $\left\{G_{1}, \ldots, G_{n}, \rho q-1\right\}$, and $\mathcal{G}$ be $a$ reduced Gröbner basis of $\mathrm{I}$ w.r.t. the lex order with $\rho>t_{r}>\cdots>t_{1}$. Let $\left\{g_{1}\left(t_{1}\right)\right\}=\mathcal{G} \cap \mathbb{K}(\bar{h})\left[t_{1}\right]$, then the square-free part of $g_{1}$ and of $S_{0}$ are equal, up to multiplication by a non-zero element in $\mathbb{K}$.

Proof. First we observe that I is zero dimensional, since its variety over $\mathbb{F}$ is $\mathfrak{F}_{\mathcal{P}}(\bar{h})$. Now, the result follows from the closure theorem, the elimination property of Gröbner bases, Hilbert's Nullstellensatz, and Theorem 6.2.

In the next corollary we use the notion of $t_{i}$-regular position of an ideal of $\bar{t}$-multivariate polynomials over a field (see [16], pp. 194), that says that if I is zero-dimensional then it is in $t_{i}$ regular position if any two zeros of I, over the algebraic closure of the ground field, have different $t_{i}$-coordinate. As commented in [16], we observe that nearly every linear change of coordinates will set the ideal in regular position.

Corollary 6.4. Let $\mathrm{I}$ be as in Corollary 6.3. If $\mathrm{I}$ is $t_{1}$-regular and radical then

$$
\operatorname{deg}\left(\Phi_{\mathcal{P}}\right)=\operatorname{deg}_{t_{1}}\left(\frac{S_{0}}{\operatorname{gcd}\left(S_{0}, \frac{\partial S_{0}}{\partial t_{1}}\right)}\right) .
$$


Proof. It follows from Corollary 6.3 and the Shape Lemma (see Theorem 8.4.6 in [16], pp. 195; observe that the notion used in this paper of reduced Gröbner basis is the notion of normed reduced Gröbner basis used in [16]).

Example. Through this example, we illustrate the results in this section. We consider the 3-dimensional rational variety $\mathcal{V} \subset \mathbb{C}^{5}$ (so, $n=5$ and $r=3$ ) given by the parametrization

$$
\mathcal{P}(\bar{t})=\left(\frac{t_{3}}{t_{2}-t_{3}}, \frac{\left(t_{1}+t_{3}\right)^{4}}{t_{2}-t_{3}}, \frac{\left(t_{1}+t_{3}\right)^{2}}{t_{2}-t_{3}}, \frac{t_{3}{ }^{4}}{t_{2}-t_{3}}, \frac{t_{3}{ }^{3}}{t_{2}-t_{3}}\right),
$$

that satisfies our assumptions. The polynomials $G_{i}$ are

$$
\begin{aligned}
G_{1}(\bar{t}, \bar{h}) & =t_{3}\left(h_{2}-h_{3}\right)-h_{3}\left(t_{2}-t_{3}\right) \\
G_{2}(\bar{t}, \bar{h}) & =\left(t_{1}+t_{3}\right)^{4}\left(h_{2}-h_{3}\right)-\left(h_{1}+h_{3}\right)^{4}\left(t_{2}-t_{3}\right) \\
G_{3}(\bar{t}, \bar{h}) & =\left(t_{1}+t_{3}\right)^{2}\left(h_{2}-h_{3}\right)-\left(h_{1}+h_{3}\right)^{2}\left(t_{2}-t_{3}\right) \\
G_{4}(\bar{t}, \bar{h}) & =t_{3}{ }^{4}\left(h_{2}-h_{3}\right)-h_{3}{ }^{4}\left(t_{2}-t_{3}\right) \\
G_{5}(\bar{t}, \bar{h}) & =t_{3}{ }^{3}\left(h_{2}-h_{3}\right)-h_{3}{ }^{3}\left(t_{2}-t_{3}\right) \\
G(\bar{t}, \bar{h}, \bar{Z}) & =G_{2}+Z_{1} G_{3}+Z_{2} G_{4}+Z_{3} G_{5} .
\end{aligned}
$$

[Base Points] We analyze the base points. For this purpose, we consider the ideal $\mathrm{I}$, in $\mathcal{C}[\bar{t}]$, generated by $\left\{p_{1}, \ldots, p_{5}, q\right\}$, and we take the Gröbner basis $\mathcal{G}$ of I w.r.t. the lex order with $t_{3}>t_{2}>t_{1}$ :

$$
\mathcal{G}=\left\{t_{1}^{2}, t_{2}, t_{3}\right\}
$$

Thus,

$$
\mathfrak{B}(\mathcal{P})=\{(0,0,0)\}
$$

and, hence, $\operatorname{dim}(\mathfrak{B}(\mathcal{P}))=0$. Therefore, $\mathfrak{H}_{2}=\mathfrak{M}_{2}^{I}=\mathfrak{L}_{2}^{I}=\emptyset$ and $\mathfrak{L}_{2}^{P}=\mathfrak{L}_{2}=\{(0,0)\}$.

[Fibre] We deal now with $\mathfrak{F}_{\mathcal{P}}(\bar{h})$. For this, we consider the ideal J, in $\mathbb{C}(\bar{h})[\rho, \bar{t}]$, generated by $\left\{G_{1}, \ldots, G_{5}, \rho q-1\right\}$, and we take the Gröbner basis $\mathcal{F}$ of J w.r.t. the lex order with $\rho>t_{3}>\cdots>$ $t_{1}$ :

$$
\mathcal{F}=\left\{-h_{1}^{2}+2 t_{1} h_{3}+t_{1}^{2}-2 h_{1} h_{3},-h_{2}+t_{2}, t_{3}-h_{3},-1+\left(h_{2}-h_{3}\right) \rho\right\} .
$$

One can check that $\mathrm{J}$ as ideal in $\mathbb{C}(\bar{h})[\rho, \bar{t}]$ is radical and $t_{1}$-regular. From $\mathcal{F}$, we get that

$$
\mathfrak{F}_{\mathcal{P}}(\bar{h})=\left\{\left(h_{1}, h_{2}, h_{3}\right),\left(-h_{1}-2 h_{3}, h_{2}, h_{3}\right)\right\} .
$$

Thus

$$
\pi_{2}\left(\mathfrak{F}_{\mathcal{P}}(\bar{h})\right)=\left\{\left(h_{1}, h_{2}\right),\left(-h_{1}-2 h_{3}, h_{2}\right\}\right.
$$

and,

$$
\left(\mathfrak{F}_{\mathcal{P}}\right)_{2}^{P}=\pi_{2}\left(\mathfrak{F}_{\mathcal{P}}(\bar{h})\right),\left(\mathfrak{F}_{\mathcal{P}}\right)_{2}^{I}=\emptyset .
$$

[Varieties associated to $\operatorname{GRS}(\mathcal{P})$ ] Since $r=3$, we only analyze $\mathfrak{M}_{2}=\mathfrak{M}_{2}^{P}$; recall that $\mathfrak{R}_{2}=\mathfrak{M}_{2}$ and that $\mathfrak{C}_{2}=\mathfrak{M}_{2}^{I}=\emptyset$. First we observe that $\operatorname{GRS}(\mathcal{P})$ is normal. On the other hand, the Gröbner basis of coeffs $\bar{Z}\left(S_{2}\right)$ w.r.t. lex order with $t_{2}>t_{1}$, as ideal in $\mathbb{C}(\bar{h})\left[t_{1}, t_{2}\right]$ is

$$
\mathcal{M}=\left\{-t_{2}\left(h_{2}-t_{2}\right), t_{1}^{2} h_{2}-h_{1}^{2} t_{2}-2 h_{1} h_{3} t_{2}+2 h_{3} t_{2} t_{1}\right\} .
$$

Therefore,

$$
\mathfrak{M}_{2}=\left\{(0,0),\left(h_{1}, h_{2}\right),\left(-h_{1}+2 h_{3}, h_{2}\right)\right\}
$$

that decomposes as (see Theorem 5.7)

$$
\mathfrak{M}_{2}=\mathfrak{M}_{2}^{P}=\left(\mathfrak{F}_{\mathcal{P}}\right)_{2}^{P} \cup \mathfrak{L}_{2}^{P} \cup \mathfrak{Q}_{0} \quad \text { with } \quad \mathfrak{Q}_{0}=\emptyset
$$

or as

$$
\mathfrak{R}_{2}=\mathfrak{M}_{2}=\pi_{2}(\mathfrak{B}(\mathcal{P}))^{*} \cup\left(\mathfrak{F}_{\mathcal{P}}\right)_{2}^{P}
$$


[Connection to Gröbner bases] The polynomials $S_{1}$ and $S_{0}$ are

$$
\begin{aligned}
& S_{1}\left(t_{1}, t_{2}\right)=t_{1}^{2}\left(-t_{1}+h_{1}\right)\left(h_{1}+2 h_{3}+t_{1}\right) \\
& S_{0}\left(t_{1}\right)=\left(-t_{1}+h_{1}\right)\left(h_{1}+2 h_{3}+t_{1}\right) .
\end{aligned}
$$

On the other hand, the univariate polynomial (in $t_{1}$ ) of the ideal of the fibre, namely J, is (compare to Corollary 6.3)

$$
-h_{1}^{2}+2 t_{1} h_{3}+t_{1}^{2}-2 h_{1} h_{3}=-S_{0}\left(t_{1}\right),
$$

and $\operatorname{deg}\left(\Phi_{\mathcal{P}}\right)=\operatorname{deg}_{t_{1}}\left(S_{0}\right)=2($ see Corollary 6.4).

\section{Appendix}

In this section we give the details of some technical proofs in the paper. More precisely, of the proofs of Lemmas 3.3, 4.2, 5.3 and 6.1, and Theorems 4.3, 5.4 and 5.7.

\section{[Proof of Lemma 3.3]}

1. Let I be the ideal of $\mathbb{K}(\bar{h})[\rho, \bar{t}]$, generated by $\mathcal{A}(\bar{h}, \bar{t}, \rho)=\left\{G_{1}, \ldots, G_{n}, q \rho-1\right\}$, where $\rho$ is a new variable. Since $\mathfrak{F}_{\mathcal{P}}(\bar{h})=\left(\mathcal{W}_{1}^{\bar{h}} \cap \cdots \cap \mathcal{W}_{n}^{\bar{h}}\right) \backslash \mathfrak{B}(\mathcal{P})$, I is zero-dimensional. Let $\mathcal{G}(\bar{h}, \bar{t}, \rho)$ be a reduced Gröbner basis of I w.r.t. the lex order with $\rho>t_{r}>\cdots>t_{1}$; reduced in the sense of Definition 5 in [8], pp. 90. There exists an open subset $\Sigma$ of $\mathbb{K}^{r}$ such that for $\bar{h}^{0} \in \Sigma, \mathcal{G}\left(\bar{h}^{0}, \bar{t}, \rho\right)$ is the Gröbner basis of $\mathcal{A}\left(\bar{h}^{0}, \bar{t}, \rho\right)$, see e.g. Example 7 in [8], pp. 283. Moreover, since I is zerodimensional, $\mathcal{G}(\bar{h}, \bar{t}, \rho) \cap \mathbb{K}(\bar{h})\left[t_{1}\right]=\left\{g\left(\bar{h}, t_{1}\right)\right\}$ and every solution of $g$ over $\mathbb{F}$ can be continued to a solution of the full system (see e.g. [16], pp. 194). Now, let $\bar{\alpha}=\left(a_{1}, \ldots, a_{n}\right) \in \mathfrak{F}_{\mathcal{P}}(\bar{h})$ be such that al least one $a_{i}$ is constant, say $a_{1} \in \mathbb{K}$. Let $\mathcal{W}^{*}$ be the algebraic set generated, over $\mathbb{K}$, by $\mathcal{P}\left(a_{1}, t_{2}, \ldots, t_{r}\right)$. Note that $\operatorname{dim}\left(\mathcal{W}^{*}\right)<r$, and that $t_{1}-a_{1}$ divides $g\left(\bar{h}, t_{1}\right)$. We consider the open set $\Omega(\mathcal{P}) \cap \Sigma$. Let $\bar{h}^{0} \in \Omega(\mathcal{P}) \cap \Sigma$. Then, $\mathcal{P}\left(\bar{h}^{0}\right)$ is well defined, and $\mathcal{G}\left(\bar{h}^{0}, \bar{t}, \rho\right)$ is a Gröbner basis of $\mathcal{A}\left(\bar{h}^{0}, \bar{t}, \rho\right)$. Since $g\left(\bar{h}^{0}, a_{1}\right)=0, a_{1}$ is extended to a solution $\left(A, \rho^{0}\right)$ of the full system, where the first component of $A$ is $a_{1}$. Therefore, $\mathcal{P}\left(\bar{h}^{0}\right)=\mathcal{P}(A)$ and hence $\mathcal{P}(\Omega(\mathcal{P}) \cap \Sigma) \subset \mathcal{W}^{*}$ which is a contradiction.

2. It is a direct consequence of the triangular structure of the reduced Gröbner basis, w.r.t. the lex order, of a zero-dimensional ideal $\mathbb{K}$-definable.

3. It follows from statement 2.

4. Let $\mathcal{W}_{1}^{\bar{\alpha}} \cap \cdots \cap \mathcal{W}_{n}^{\bar{\alpha}}$ contain a hypersurface in $\mathbb{K}^{r}$, and $M(\bar{t})$ its defining polynomial. Then, there exist $N_{i} \in \mathbb{K}[\bar{t}]$ such that

$$
G_{i}(\bar{t}, \bar{\alpha})=p_{i}(\bar{t}) q(\bar{\alpha})-p_{i}(\bar{\alpha}) q(\bar{t})=M(\bar{t}) N_{i}(\bar{t}), \text { for } i=1, \ldots, n .
$$

Observe that, since $\operatorname{gcd}\left(p_{1}, \ldots, p_{n}, q\right)=1$, then $\operatorname{gcd}(q(\bar{t}), M(\bar{t}))=1$. Now, we consider the set $\Lambda_{\bar{\alpha}}:=\left\{\bar{\beta} \in \mathbb{K}^{n} / M(\bar{\beta})=0, q(\bar{\beta}) \neq 0\right\} . \Lambda_{\bar{\alpha}} \neq \emptyset$ is an open subset of $\mathbb{V}_{\mathbb{K}}(M)$. Moreover $\Lambda_{\bar{\alpha}} \subset \mathfrak{F}_{\mathcal{P}}(\bar{\alpha})$, which is impossible since $\operatorname{card}\left(\Lambda_{\bar{\alpha}}\right)=\infty$ and $\mathfrak{F}_{\mathcal{P}}(\bar{\alpha})$ is zero dimensional because $\bar{\alpha} \in \Omega(\mathcal{P})$.

5. This statement follows from statement 4 .

\section{[Proof of Lemma 4.2]}

1. Let $M=M_{2}+Z_{1} M_{3}+\cdots+Z_{\ell-2} M_{\ell}$, and let $\mathcal{R}$ be the set of all the roots of $M_{1}$ in the algebraic closure of the quotient field of $\mathbb{L}$. The result follows from

$$
0 \neq \operatorname{res}_{x}\left(M_{1}, M\right)=\operatorname{LCoeff}\left(M_{1}\right)^{\operatorname{deg}_{x}(M)} \prod_{\alpha \in \mathcal{R}} M(\alpha) .
$$

2. Since $M$ does not have factors in $\mathbb{L}[x]$ then $\operatorname{gcd}\left(M\left(\Delta^{*}, x\right), M(\Delta, x)\right)=1$, and hence $N \neq 0$. Let $\mathcal{T}$ be the set of non-constant monomials in $\Delta$ appearing in $M$. We express $M$ as $M(\Delta, x)=$ 
$a(x)+\sum_{T \in \mathcal{T}} a_{T}(x) T$. If $\mathcal{R}$ is the set of all roots of $M\left(\Delta^{*}, x\right)$, in the algebraic closure of the quotient field of $\mathbb{L}\left[\Delta^{*}\right]$, as univariate polynomial in $x$, then

$$
N=\operatorname{LCoeff}\left(M\left(\Delta^{*}, x\right), x\right)^{\operatorname{deg}_{x}(M)} \prod_{\alpha \in \mathcal{R}} M(\Delta, \alpha) .
$$

Since $N \neq 0$, if $N$ does not depend on $\Delta$, then $a_{T}(\alpha)=0$ for all $T \in \mathcal{T}$ and for all $\alpha \in \mathcal{R}$. So, $M(\Delta, x)-a(x)=B\left(\Delta, \Delta^{*}, x\right) M\left(\Delta^{*}, x\right)$ for some polynomial $B$. If $a=0, M\left(\Delta^{*}, x\right)$ divides $M(\Delta, x)$. Thus $M\left(\Delta^{*}, x\right) \in \mathbb{L}[x]$, and hence $M(\Delta, x) \in \mathbb{L}[x]$ which is a contradiction. If $a \neq 0$ then $M\left(\Delta^{*}, x\right)\left(1-B\left(\Delta^{*}, \Delta^{*}, x\right)\right)=a(x)$. So, $M$ divides $a$ which is again a contradiction. So $N$ depends on $\Delta$, and reasoning similarly we get that also depends on $\Delta^{*}$. For the second part, let $C\left(\Delta^{*}\right)$ be a factor of $N$ depending only on $\Delta^{*}$; similarly if it only depends on $\Delta$. Let $P$ be a solution of $C$ over the algebraic closure $\mathbb{M}$ of the quotient field of $\mathbb{L}$. Then, $N(P, \Delta)=0$ and since LCoeff $(M, x)(\Delta) \neq 0$ there exists $a \in \mathbb{M}$ such that $M(P, a)=0=M(\Delta, a)=0$. This implies that $(x-a)$ divides $M$, which is a contradiction.

\section{[Proof of Theorem 4.3]}

We prove statements 1 and 2 simultaneously. We start with the case $i=r-1$. By Lemma 3.3, statement $5, \operatorname{gcd}\left(G_{1}, \ldots, G_{r}\right)=1$, and by Proposition 2.2, we deduce that $G_{1}$ is not constant. Therefore, by assumption A-3, and Lemma 4.2, statement 1, applied to $R_{r-1}$, we get that $R_{r-1}$ depends on $\bar{Z}$ and so $S_{r-1}$ does. Moreover, by definition, $S_{r-1}$ does not have factors in $\mathbb{K}[\bar{t}, \bar{h}]$. Now, for $i=r-2$, applying Lemma 4.2, statement 2 , to $S_{r-1}$, and taking $\mathbb{L}=\mathbb{K}\left[\bar{t}^{r-2}, \bar{h}\right]$ and $\Delta=\bar{Z}$, one gets the result. Similarly, for $R_{i}$ with $i<r-2$ and for $S_{i}$ with $2<i<r-2$. Finally, since $R_{1}$ is not zero it follows that $S_{1}, S_{0}$ are not zero either.

To prove statement 3, let us assume that $S_{r-1}$ does not depend on $\bar{t}$. By statement 2, $S_{r-1}$ depends on $\bar{Z}$. Let $\bar{\alpha} \in \mathbb{F}^{r-2}$ be such that $S_{r-1}$ vanishes at $\bar{Z}=\bar{\alpha}$. By Proposition 3.1, there exists $a$ such that $G_{1}\left(\bar{t}^{r-1}, a, \bar{h}, \bar{\alpha}\right)=G\left(\bar{t}^{r-1}, a, \bar{h}, \bar{\alpha}\right)=0$. Moreover, since $G_{1}\left(\bar{t}^{r-1}, t_{r}, \bar{h}, \bar{\alpha}\right) \in$ $\mathbb{F}\left[\bar{t}^{r-1}\right]\left[t_{r}\right]$, one has that $a$ does not depends on $\bar{Z}$, and hence $G_{i}\left(\bar{t}^{r-1}, a, \bar{h}, \bar{\alpha}\right)=0$ for $i=$ $1, \ldots, n$. Let us see that $q\left(\bar{t}^{r-1}, a\right) \neq 0$. Indeed, if it vanishes, then $p_{i}\left(\bar{t}^{r-1}, a\right)=0$ for $i=$ $1, \ldots, n$. Therefore $\left(t_{r}-a\right)$ divides to the $\operatorname{gcd}\left(p_{1}, \ldots, p_{n}, q\right)$ which is a contradiction. This implies that $\mathcal{P}(\bar{t})=\mathcal{P}\left(\bar{t}^{r-1}, a\right)$. But this is impossible because $a$ belongs to the algebraic closure of $\mathbb{F}\left[\bar{t}^{r-1}\right]$ and hence it does not depend on $t_{r}$ while $\mathcal{P}(\bar{t})$ does since $\operatorname{dim}(\mathcal{V})=r$.

\section{[Proof of Lemma 5.3]}

The right-left inclusion is clear. We prove $\mathfrak{M}_{j}^{P} \subset\left(\mathfrak{M}_{j}^{P} \cap \pi_{j}\left(\mathfrak{F}_{\mathcal{P}}(\bar{h})\right)\right) \cup\left(\mathfrak{M}_{j}^{P} \cap \mathfrak{L}_{j}^{P}\right)$ by induction. We start with $j=r-1$. let $P \in \Omega=\mathfrak{M}_{r-1}^{P} \backslash \mathfrak{H}_{r-1}$; note that, since no component of $\mathfrak{M}_{r-1}^{P}$ is included in $\mathfrak{H}_{r-1}^{P}, \Omega^{*}=\mathfrak{M}_{r-1}^{P}$. Then, $S_{r-1}(P)=0$ and hence $R_{r-1}(P)=0$. By Proposition 3.1, statement 2, $P$ extends to a common solution $P^{*}$ of $\left\{G_{1}, G\right\}$. Moreover, since $P \in \mathbb{F}_{r-1}^{r-1}$ and $G_{1}\left(P, t_{r}, \bar{h}\right) \in$ $\mathbb{F}_{r-1}\left[t_{r}\right]$ one gets that $P^{*} \in \mathbb{F}_{r-1}^{r}$. Therefore, $G\left(P^{*}, \bar{h}, \bar{Z}\right)=0$ implies that $G_{i}\left(P^{*}, \bar{h}\right)=0$ for all $i=1, \ldots, n$. Thus, $P^{*} \in \mathcal{W}_{1}^{\bar{h}} \cap \cdots \cap \mathcal{W}_{n}^{\bar{h}}=\mathfrak{F}_{\mathcal{P}}(\bar{h}) \cup \mathfrak{B}(\mathcal{P})$. So, $P \in \pi_{r-1}\left(\mathfrak{F}_{\mathcal{P}}(\bar{h})\right) \cup$ $\pi_{r-1}(\mathfrak{B}(\mathcal{P})) \subset \pi_{r-1}\left(\mathfrak{F}_{\mathcal{P}}(\bar{h})\right) \cup \pi_{r-1}(\mathfrak{B}(\mathcal{P}))^{*}=\pi_{r-1}\left(\mathfrak{F}_{\mathcal{P}}(\bar{h})\right) \cup \mathfrak{L}_{r-1} \cup \mathfrak{H}_{r-1}$. But $P \notin \mathfrak{H}_{r-1}$ and hence $P \in \pi_{r-1}\left(\mathfrak{F}_{\mathcal{P}}(\bar{h})\right) \cup \mathfrak{L}_{r-1}^{P}$. Thus, $\Omega \subset\left(\mathfrak{M}_{r-1}^{P} \cap \pi_{r-1}\left(\mathfrak{F}_{\mathcal{P}}(\bar{h})\right)\right) \cup\left(\mathfrak{M}_{r-1}^{P} \cap \mathfrak{L}_{r-1}^{P}\right)$. Therefore, $\mathfrak{M}_{r-1}^{P}=\Omega^{*} \subset\left(\mathfrak{M}_{r-1}^{P} \cap \pi_{r-1}\left(\mathfrak{F}_{\mathcal{P}}(\bar{h})\right)\right) \cup\left(\mathfrak{M}_{r-1}^{P} \cap \mathfrak{L}_{r-1}^{P}\right)$.

Now, let the inclusion hold for $j$ and we prove it for $j-1$. Let $\Omega=\mathfrak{M}_{j-1}^{P} \backslash\left(\pi_{j-1}\left(\mathfrak{M}_{j}^{I}\right)^{*} \cup\right.$ $\left.\mathfrak{H}_{j-1}\right)$. As above, note that $\Omega^{*}=\mathfrak{M}_{j-1}^{P}$. Let $P \in \Omega$. Then, $S_{j-1}(P)=0$ and hence $R_{j-1}(P)=0$. By the normality assumption, $P$ extends to a common solution $P^{*}$ of

$$
\left\{S_{j}\left(\bar{t}^{j}, \bar{h}, \bar{W}_{r-2}, \ldots, \bar{W}_{j-1}\right), S_{j}\left(\bar{t}^{j}, \bar{h}, \bar{W}_{r-2}, \ldots, \bar{W}_{j}, \bar{Z}\right)\right\}
$$


if $j=r-2$ then $\left\{S_{r-1}\left(\bar{t}^{r-1}, \bar{h}, \bar{W}_{r-2}\right), S_{r-1}\left(\bar{t}^{r-1}, \bar{h}, \bar{Z}\right)\right\}$. Moreover, since $P \in \mathbb{F}_{j-1}^{j-1}$ and $S_{j}\left(P, t_{j}, \bar{h}, \bar{W}_{r-2}, \ldots, \bar{W}_{j-1}\right) \in \mathbb{F}_{j-1}\left[t_{j}\right]$ one gets that $P^{*} \in \mathbb{F}_{j-1}^{j}$. Therefore,

$$
S_{j}\left(P^{*}, \bar{h}, \bar{W}_{r-2}, \ldots, \bar{W}_{j}, \bar{Z}\right)=0
$$

implies that $P^{*} \in \mathfrak{M}_{j}$. Moreover, by construction, $P \notin \pi_{j-1}\left(\mathfrak{M}_{j}^{I}\right)^{*}$ and hence $P^{*} \notin \mathfrak{M}_{j}^{I}$. Thus, $P^{*} \in \mathfrak{M}_{j}^{P}$. Now, by the induction hypothesis, $P^{*} \in\left(\mathfrak{M}_{j}^{P} \cap \pi_{j}\left(\mathfrak{F}_{\mathcal{P}}(\bar{h})\right)\right) \cup\left(\mathfrak{M}_{j}^{P} \cap \mathfrak{L}_{j}^{P}\right)$. So $P^{*} \in$ $\pi_{j}\left(\mathfrak{F}_{\mathcal{P}}(\bar{h})\right) \cup \mathfrak{L}_{j}^{P}$. Therefore, $P \in \pi_{j-1}\left(\mathfrak{F}_{\mathcal{P}}(\bar{h})\right) \cup \pi_{j-1}\left(\mathfrak{L}_{j}^{P}\right) \subset \pi_{j-1}\left(\mathfrak{F}_{\mathcal{P}}(\bar{h})\right) \cup \pi_{j-1}\left(\mathfrak{L}_{j}^{P}\right)^{*}=$ $\pi_{j-1}\left(\mathfrak{F}_{\mathcal{P}}(\bar{h})\right) \cup \mathfrak{L}_{j-1} \cup \mathfrak{H}_{j-1}$. Now, since $P \notin \pi_{j-1}\left(\mathfrak{M}_{j}^{I}\right)^{*} \cup \mathfrak{H}_{j-1}$, the proof follows as above.

\section{[Proof of Theorem 5.4]}

We prove statements 1 and 2 simultaneously. Let $\operatorname{cont} \bar{Z}_{Z}\left(R_{j}\right)$ have a factor $M$ depending on $\bar{t}^{j}$; assume w.l.o.g. that $M$ is irreducible. Let $\mathcal{A}=\mathbb{V}_{\mathbb{F}_{j}}(M) \subset \mathbb{F}_{j}^{j}$, and $\Omega=\mathcal{A} \backslash \pi_{j}\left(\mathfrak{F}_{\mathcal{P}}(\bar{h})\right)$. Since $\operatorname{dim}\left(\mathfrak{F}_{\mathcal{P}}(\bar{h})\right)=0, \Omega \neq \emptyset$ and open in $\mathcal{A}$. Thus, $\operatorname{dim}(\Omega)$ is $r-2$ for statement 1 , and $j-1$ for statement 2 . Since $\operatorname{GRS}(\mathcal{P})$ is normal, every point in $P \in \Omega$ extends to a common solution $P^{*}$ of $\left\{G_{1}, G\right\}$ (for statement 1) or of

$$
\left\{S_{j+1}\left(\bar{t}^{j+1}, \bar{h}, \bar{W}_{r-2}, \ldots, \bar{W}_{j}\right), S_{j+1}\left(\bar{t}^{j+1}, \bar{h}, \bar{W}_{r-2}, \ldots, \bar{W}_{j+1}, \bar{Z}\right)\right\}
$$

(for statement 2); if $j=r-2$ then $\left\{S_{r-1}\left(\bar{t}^{r-1}, \bar{h}, \bar{W}_{r-2}\right), S_{r-1}\left(\bar{t}^{r-1}, \bar{h}, \bar{Z}\right)\right\}$. Say that $\Omega^{e}$ is the set of extended common solutions. For every $P \in \Omega \subset \mathbb{F}^{j}{ }^{j}$, since $G_{1}\left(P, t_{r}, \bar{h}\right) \in \mathbb{F}\left[t_{r}\right]$ (for statement 1) and $S_{j+1}\left(P, t_{j+1}, \bar{h}, \bar{W}_{r-2}, \ldots, \bar{W}_{j}\right) \in \mathbb{F}_{j}\left[t_{j+1}\right]$ (for statement 2), its extension $P^{*}$ belongs to $\mathbb{F}^{r}$ (for statement 1) or to $\mathbb{F}_{j}^{j+1}$ (for statement 2). Therefore, $\Omega^{e} \subset \mathbb{F}^{r}$ (for statement 1) and $\Omega^{e} \subset \mathbb{F}_{j}^{j+1}$ (for statement 2). Thus, $\Omega^{e} \subset\left(\mathcal{W}_{1}^{\bar{h}} \cap \cdots \cap \mathcal{W}_{n}^{\bar{h}}\right) \backslash \mathfrak{F}_{\mathcal{P}}(\bar{h})=\mathfrak{B}(\mathcal{P})$ (for statement 1) or, by Lemma 5.3, $\Omega^{e} \subset \mathfrak{M}_{j+1} \backslash \pi_{j+1}\left(\mathfrak{F}_{\mathcal{P}}(\bar{h})\right)=\left(\mathfrak{M}_{j+1}^{P} \backslash \pi_{j+1}\left(\mathfrak{F}_{\mathcal{P}}(\bar{h})\right) \cup\left(\mathfrak{M}_{j+1}^{I} \backslash \pi_{j+1}\left(\mathfrak{F}_{\mathcal{P}}(\bar{h})\right)=\right.\right.$ $\left[\left(\left(\mathfrak{M}_{j+1}^{P} \cap \pi_{j+1}\left(\mathfrak{F}_{\mathcal{P}}(\bar{h})\right) \cup\left(\mathfrak{M}_{j+1}^{P} \cap \mathfrak{L}_{j+1}^{P}\right)\right) \backslash \pi_{j+1}\left(\mathfrak{F}_{\mathcal{P}}(\bar{h})\right)\right] \cup\left(\mathfrak{M}_{j+1}^{I} \backslash \pi_{j+1}\left(\mathfrak{F}_{\mathcal{P}}(\bar{h})\right)=\left[\left(\mathfrak{M}_{j+1}^{P} \cap\right.\right.\right.\right.$ $\left.\left.\mathfrak{L}_{j+1}^{P}\right) \backslash \pi_{j+1}\left(\mathfrak{F}_{\mathcal{P}}(\bar{h})\right)\right] \cup\left(\mathfrak{M}_{j+1}^{I} \backslash \pi_{j+1}\left(\mathfrak{F}_{\mathcal{P}}(\bar{h})\right)\right) \subset\left(\mathfrak{M}_{j+1}^{P} \cap \mathfrak{L}_{j+1}^{P}\right) \cup \mathfrak{M}_{j+1}^{I}$ (for statement 2). Then, $\left.\Omega \subset \pi_{j}\left(\mathfrak{M}_{j+1} \cap \pi_{j+1}(\mathfrak{B}(\mathcal{P}))\right)^{*}\right)$, and hence $r-2=\operatorname{dim}(\Omega) \leq \operatorname{dim}\left(\pi_{r-1}(\mathfrak{B}(\mathcal{P}))\right.$ ) (for statement 1) and $\left.j-1=\operatorname{dim}(\Omega) \leq \operatorname{dim}\left(\pi_{j}\left(\left(\mathfrak{M}_{j+1}^{P} \cap \mathfrak{L}_{j+1}^{P}\right) \cup \mathfrak{M}_{j+1}^{I}\right)\right)\right)$ (for statement 2); which is a contradiction.

In order to prove statement 3 , let $H \in \mathbb{K}\left[\bar{t}^{r-1}\right]$ be the defining polynomial of $\mathfrak{H}_{r-1} . G_{1}$ and $G$ vanish on $\mathfrak{B}(\mathcal{P})$, so $R_{r-1}$ vanishes on $\pi_{r-1}(\mathfrak{B}(\mathcal{P}))$; in particular on $\mathfrak{H}_{r-1}$. Furthermore, since $\mathfrak{H}_{r-1} \subset \mathbb{F}^{r-1}$, all coefficients of $R_{r-1}$ w.r.t. $\bar{Z}$ vanish on $\mathfrak{H}_{r-1}$. Thus, $\mathfrak{H}_{r-1} \subset \mathfrak{R}_{r-1}$. Furthermore, since $\operatorname{dim}\left(\mathfrak{H}_{r-1}\right)=r-2, \mathfrak{H}_{r-1} \subset \mathfrak{C}_{r-1}$. Now, let us assume that $\operatorname{cont}_{\bar{Z}}\left(R_{r-1}\right)$ has another factor $H^{*}$, coprime with $H$, and depending on $\bar{t}^{r-1}$. Let $\mathfrak{H}_{r-1}^{*}=\mathbb{V}_{\mathbb{F}}\left(H^{*}\right)$. We take $\Omega=\mathfrak{H}_{r-1}^{*} \backslash \pi_{r-1}(\mathfrak{B}(\mathcal{P}))^{*}=\mathfrak{H}_{r-1}^{*} \backslash\left(\mathfrak{H}_{r-1} \cup \mathfrak{L}_{r-1}\right)$. Since $\mathfrak{H}_{r-1}^{*} \neq \mathfrak{H}_{r-1}, \operatorname{dim}(\Omega)=r-2$. Every $P \in \Omega$, by the normality, extends to $P^{*} \in \mathcal{W}_{1}^{\bar{h}} \cap \cdots \cap \mathcal{W}_{n}^{\bar{h}}=\mathfrak{F}_{\mathcal{P}}(\bar{h}) \cup \mathfrak{B}(\mathcal{P})$; note that $P$ does not depend on $\bar{Z}$ and $G_{1} \in \mathbb{F}[\bar{t}]$. Let $\Omega^{e} \subset \mathfrak{F}_{\mathcal{P}}(\bar{h}) \cup \mathfrak{B}(\mathcal{P})$ be the set of extended points from $\Omega \cdot \operatorname{dim}\left(\Omega^{e}\right) \geq r-2$. Therefore, since $r>2$ and $\operatorname{dim}(\mathfrak{B}(\mathcal{P}))=r-2, \Omega^{e} \cap \mathfrak{B}(\mathcal{P}) \neq \emptyset$. Let $P^{*} \in \Omega^{e} \cap \mathfrak{B}(\mathcal{P})$. Thus, $\pi_{r-1}\left(P^{*}\right) \in \Omega \cap \pi_{r-1}(\mathfrak{B}(\mathcal{P}))=\emptyset$, which is a contradiction. Therefore, $\mathfrak{H}_{r-1}=\mathfrak{C}_{r-1}$.

To prove statement 4 , let $\mathfrak{Q}$ be the hypersurface included in $\pi_{j}\left(\left(\mathfrak{M}_{j+1}^{P} \cap \mathfrak{L}_{j+1}^{P}\right) \cup \mathfrak{M}_{j+1}^{I}\right)^{*}$ and let $H$ be its defining polynomial.

$$
S_{j+1}\left(\bar{t}^{j+1}, \bar{h}, \bar{W}_{r-2}, \ldots, \bar{W}_{j}\right), S_{j+1}\left(\bar{t}^{j+1}, \bar{h}, \bar{W}_{r-2}, \ldots, \bar{W}_{j+1}, \bar{Z}\right)
$$

vanish on $\mathfrak{M}_{j+1}$; in particular, $R_{j}$ vanishes on $\mathfrak{Q} \subset \pi_{j}\left(\mathfrak{M}_{j+1}\right)^{*}$. Furthermore, since $\mathfrak{Q} \subset \mathbb{F}_{j}^{j}$, all coefficients of $R_{j}$ w.r.t. $\bar{Z}$ vanish on $\mathfrak{Q}$. Thus, $\mathfrak{Q} \subset \mathfrak{R}_{j}$. Furthermore, since $\operatorname{dim}(\mathfrak{Q})=j-1$, $\mathfrak{Q} \subset \mathfrak{C}_{j}$. Now, let us assume that $\operatorname{cont}_{\bar{Z}}\left(R_{j}\right)$ has another factor $H^{*}$, coprime with $H$, and depending on $\bar{t}^{j}$; say that $\mathfrak{T}=\mathbb{V}_{\mathbb{F}_{j}}\left(H^{*}\right)$. We consider the non-empty open set $\Omega=\mathfrak{T} \backslash\left(\mathfrak{Q} \cup \pi_{j}\left(\mathfrak{F}_{\mathcal{P}}\right)\right)$; note that $\operatorname{dim}\left(\pi_{j}\left(\mathfrak{F}_{\mathcal{P}}\right)\right)=0$ and that $\operatorname{gcd}\left(H, H^{*}\right)=1$. Every $P \in \Omega$ extends to a common solution $P^{*}$ of 
$S_{j+1}\left(\bar{t}^{j+1}, \bar{h}, \bar{W}_{r-2}, \ldots, \bar{W}_{j}\right)$ and $S_{j+1}\left(\bar{t}^{j+1}, \bar{h}, \bar{W}_{r-2}, \ldots, \bar{W}_{j+1}, \bar{Z}\right)$. Since $P$ does not depend on $\bar{Z}, P^{*} \in \mathfrak{M}_{j+1}$. By construction $P^{*} \notin \pi_{j+1}\left(\mathfrak{F}_{\mathcal{P}}\right)$. Thus, by Lemma 5.3, $P^{*} \in\left(\mathfrak{M}_{j+1}^{P} \cap\right.$ $\left.\mathfrak{L}_{j+1}^{P}\right) \cap \mathfrak{M}_{j+1}^{I}$. Therefore, if we denote by $\Omega^{e}$ the set of extended solutions of $\Omega, \Omega^{e} \subset\left(\mathfrak{M}_{j+1}^{P} \cap\right.$ $\left.\mathfrak{L}_{j+1}^{P}\right) \cap \mathfrak{M}_{j+1}^{I}$. Furthermore, $\Omega=\pi_{j}\left(\Omega^{e}\right) \subset \pi_{j}\left(\left(\mathfrak{M}_{j+1}^{P} \cap \mathfrak{L}_{j+1}^{P}\right) \cap \mathfrak{M}_{j+1}^{I}\right)$. But $\operatorname{dim}(\Omega)=j-1$, and hence $\Omega \subset \mathfrak{Q}$ which is a contradiction.

\section{[Proof of Theorem 5.7]}

Statements 1 and 2 follow from the normality and from the fact that the involved points do not depend on $\bar{Z}$.

We prove statement 3 by induction. $\mathfrak{R}_{r-1}=\mathfrak{C}_{r-1} \cup \mathfrak{M}_{r-1}^{P} \cup \mathfrak{M}_{r-1}^{I}$. By Theorem 5.4, $\mathfrak{C}_{r-1}=\mathfrak{H}_{r-1}$ and, by statement $1, \mathfrak{R}_{r-1}=\pi_{r-1}(\mathfrak{B}(\mathcal{P}))^{*} \cup\left(\mathfrak{F}_{\mathcal{P}}\right)_{r-1}^{P}=\mathfrak{H}_{r-1} \cup \mathfrak{L}_{r-1} \cup\left(\mathfrak{F}_{\mathcal{P}}\right)_{r-1}^{P}=\mathfrak{H}_{r-1} \cup$ $\mathfrak{L}_{r-1}^{P} \cup\left(\mathfrak{F}_{\mathcal{P}}\right)_{r-1}^{P}$. Thus, $\mathfrak{H}_{r-1} \cup \mathfrak{M}_{r-1}^{P} \cup \mathfrak{M}_{r-1}^{I}=\mathfrak{H}_{r-1} \cup \mathfrak{L}_{r-1}^{P} \cup\left(\mathfrak{F}_{\mathcal{P}}\right)_{r-1}^{P}$. So $\left(\left(\mathfrak{H}_{r-1} \cup \mathfrak{M}_{r-1}^{P} \cup\right.\right.$ $\left.\left.\mathfrak{M}_{r-1}^{I}\right) \backslash \mathfrak{H}_{r-1}\right)^{*}=\left(\left(\mathfrak{H}_{r-1} \cup \mathfrak{L}_{r-1}^{P} \cup\left(\mathfrak{F}_{\mathcal{P}}\right)_{r-1}^{P}\right) \backslash \mathfrak{H}_{r-1}\right)^{*}$. Therefore $\left(\mathfrak{H}_{r-1} \backslash \mathfrak{H}_{r-1}\right)^{*} \cup\left(\mathfrak{M}_{r-1}^{P} \backslash\right.$ $\left.\mathfrak{H}_{r-1}\right)^{*} \cup\left(\mathfrak{M}_{r-1}^{I} \backslash \mathfrak{H}_{r-1}\right)^{*}=\left(\mathfrak{H}_{r-1} \backslash \mathfrak{H}_{r-1}\right)^{*} \cup\left(\mathfrak{L}_{r-1}^{P} \backslash \mathfrak{H}_{r-1}\right)^{*} \cup\left(\left(\mathfrak{F}_{\mathcal{P}}\right)_{r-1}^{P} \backslash \mathfrak{H}_{r-1}\right)^{*}$. Hence, $\mathfrak{M}_{r-1}^{P}=\mathfrak{L}_{r-1}^{P} \cup\left(\mathfrak{F}_{\mathcal{P}}\right)_{r-1}^{P} \cup \mathfrak{Q}_{r-1}$, where $\mathfrak{Q}_{r-1}=\emptyset$.

Let the result be true for $j+1 \leq r-1$. By the induction hypothesis, $\left(\mathfrak{M}_{j+1}^{P} \cap \mathfrak{L}_{j+1}^{P}\right) \cup$ $\mathfrak{M}_{j+1}^{I}=\left[\left(\left(\mathfrak{F}_{\mathcal{P}}\right)_{j+1}^{P} \cup \mathfrak{L}_{j+1}^{P} \cup \mathfrak{Q}_{j+1}\right) \cap \mathfrak{L}_{j+1}^{P}\right] \cup \mathfrak{M}_{j+1}^{I}=\mathfrak{L}_{j+1}^{P} \cup\left(\mathfrak{Q}_{j+1} \cap \mathfrak{L}_{j+1}^{P}\right) \cup \mathfrak{M}_{j+1}^{I}$. Therefore, $\pi_{j}\left(\left(\mathfrak{M}_{j+1}^{P} \cap \mathfrak{L}_{j+1}^{P}\right) \cup \mathfrak{M}_{j+1}^{I}\right)^{*}=\pi_{j}\left(\mathfrak{L}_{j+1}^{P}\right)^{*} \cup \pi_{j}\left(\mathfrak{Q}_{j+1} \cap \mathfrak{L}_{j+1}^{P}\right) \cup \pi_{j}\left(\mathfrak{M}_{j+1}^{I}\right)^{*}=\mathfrak{H}_{j} \cup \mathfrak{L}_{j} \cup$ $\pi_{j}\left(\mathfrak{Q}_{j+1} \cap \mathfrak{L}_{j+1}^{P}\right) \cup \pi_{j}\left(\mathfrak{M}_{j+1}^{I}\right)^{*}$ and, by Theorem 5.4, $\mathfrak{C}_{j}$ is either empty or the hypersurface included in $\mathfrak{H}_{j} \cup \mathfrak{L}_{j} \cup \pi_{j}\left(\mathfrak{Q}_{j+1} \cap \mathfrak{L}_{j+1}^{P}\right) \cup \pi_{j}\left(\mathfrak{M}_{j+1}^{I}\right)^{*}$; that is, the hypersurface included in $\Delta=\mathfrak{H}_{j} \cup$ $\pi_{j}\left(\mathfrak{M}_{j+1}^{I}\right)^{*}$. On the other hand, by the induction hypothesis and by statement $2, \mathfrak{C}_{j} \cup \mathfrak{M}_{j}=\mathfrak{R}_{j}=$ $\pi_{j}\left(\mathfrak{M}_{j+1}\right)^{*}=\pi_{j}\left(\mathfrak{M}_{j+1}^{P} \cup \mathfrak{M}_{j+1}^{I}\right)^{*}=\pi_{j}\left(\left(\mathfrak{F}_{\mathcal{P}}\right)_{j+1}^{P} \cup \mathfrak{L}_{j+1}^{P} \cup \mathfrak{Q}_{j+1} \cup \mathfrak{M}_{j+1}^{I}\right)^{*}=\pi_{j}\left(\left(\mathfrak{F}_{\mathcal{P}}\right)_{j+1}^{P}\right) \cup$ $\pi_{j}\left(\mathfrak{L}_{j+1}^{P}\right)^{*} \cup \pi_{j}\left(\mathfrak{Q}_{j+1}\right) \cup \pi_{j}\left(\mathfrak{M}_{j+1}^{I}\right)^{*}=\pi_{j}\left(\left(\mathfrak{F}_{\mathcal{P}}\right)_{j+1}^{P}\right) \cup \mathfrak{H}_{j} \cup \mathfrak{L}_{j} \cup \pi_{j}\left(\mathfrak{Q}_{j+1}\right) \cup \pi_{j}\left(\mathfrak{M}_{j+1}^{I}\right)^{*}$. Now, we express $\pi_{j}\left(\left(\mathfrak{F}_{\mathcal{P}}\right)_{j+1}^{P}\right) \cup \pi_{j}\left(\mathfrak{Q}_{j+1}\right)$ as $\left(\mathfrak{F}_{\mathcal{P}}\right)_{j}^{P} \cup \mathfrak{Q}_{0}$ where $\mathfrak{Q}_{0} \subset\left(\mathfrak{F}_{\mathcal{P}}\right)_{j}^{I}$. Then, $\mathfrak{C}_{j} \cup \mathfrak{M}_{j}=$ $\left(\mathfrak{F}_{\mathcal{P}}\right)_{j}^{P} \cup \mathfrak{H}_{j} \cup \mathfrak{L}_{j} \cup \mathfrak{Q}_{0} \cup \pi_{j}\left(\mathfrak{M}_{j+1}^{I}\right)^{*}$. In this situation, we subtract $\Delta$ to get $\mathfrak{M}_{j} \backslash \Delta=\left(\left(\mathfrak{F}_{\mathcal{P}}\right)_{j}^{P} \backslash\right.$ $\Delta) \cup\left(\mathfrak{H}_{j} \backslash \Delta\right) \cup\left(\mathfrak{L}_{j} \backslash \Delta\right) \cup\left(\mathfrak{Q}_{0} \backslash \Delta\right) \cup\left(\pi_{j}\left(\mathfrak{M}_{j+1}^{I}\right)^{*} \backslash \Delta\right)=\left(\mathfrak{F}_{\mathcal{P}}\right)_{j}^{P} \cup\left(\mathfrak{L}_{j}^{P} \backslash \Delta\right) \cup\left(\mathfrak{Q}_{0} \backslash \Delta\right)$. Taking Zariski closures we get that $\mathfrak{M}_{j}^{P}=\left(\mathfrak{F}_{\mathcal{P}}\right)_{j}^{P} \cup \mathfrak{L}_{j}^{P} \cup \mathfrak{Q}_{j}$ with $\mathfrak{Q}_{j}=\mathfrak{Q}_{0} \backslash \Delta \subset \mathfrak{Q}_{0} \subset\left(\mathfrak{F}_{\mathcal{P}}\right)_{j}^{I}$.

[Proof of Lemma 6.1]

1. Let $P \in \mathfrak{F}_{\mathcal{P}}(\bar{h})$ and $P_{i}=\pi_{i}(P)$. The proof goes as follows: (a) we prove by induction that, for $i \in\{2, \ldots, r-1\}, P_{i} \in \mathfrak{M}_{i}$; (b) we prove that $S_{0}\left(P_{1}\right)=0$.

(a) Since $G_{i}$ vanishes at $P$, and $P$ does not depend on $\bar{Z}, P_{r-1} \in \mathfrak{R}_{r-1}=\mathfrak{C}_{r-1} \cup \mathfrak{M}_{r-1}$. So, by Corollary 5.6, $P_{r-1} \in \mathfrak{M}_{r-1}$. Now, let $P_{i} \in \mathfrak{M}_{i}$. Then $P_{i}$ does not depend on $\bar{Z}$ and, hence, $P_{i-1} \in \mathfrak{R}_{i-1}=\mathfrak{C}_{i-1} \cap \mathfrak{M}_{i-1}$. Now, by Corollary 5.6, $P_{i-1} \in \mathfrak{M}_{i-1}$.

(b) Since $P_{2} \in \mathfrak{M}_{2}, P_{2}$ does not depend on $\bar{Z}$, and hence $R_{1}\left(P_{1}\right)=0$. Moreover, since $P_{1}$ does not depend on $\bar{Z}, \bar{W}_{\ell}$, and since $R_{1}$ is univariate in $t_{1}$, one has that $S_{1}\left(P_{1}\right)=0$. Finally, since the polynomial $S_{1}$ is univariate in $t_{1}$ and $P_{1}$ does depend on $\bar{h}$ (see Lemma 3.3, statement 1), one concludes that $S_{0}\left(P_{1}\right)=0$.

2. Since $\bar{h} \in \mathfrak{F}_{\mathcal{P}}(\bar{h})$, by statement $1, S_{0}\left(h_{1}\right)=0$. Hence $S_{0}$ is not constant. Moreover, since $S_{0}$ is primitive w.r.t. $\bar{h}$, all roots of $S_{0}$ are in $\mathbb{F} \backslash \mathbb{K}$. On the other hand, by definition, $\mathfrak{L}_{2}^{P}$ is either empty or zero-dimensional. In this situation, if $S_{0}(\alpha)=0$, then $S_{1}(\alpha)=0$, and hence $R_{1}(\alpha)=0$. Moreover, by the normality assumption, $\alpha$ extends to a common solution $(\alpha, \beta)$ not depending on $\bar{Z}$. So, $(\alpha, \beta) \in \mathfrak{M}_{2}^{P}=\mathfrak{M}_{2}$ (see Remark 5.2). By Theorem 5.7, $(\alpha, \beta) \in \mathfrak{Q}_{2} \cup \mathfrak{L}_{2}^{P} \cup\left(\mathfrak{F}_{\mathcal{P}}\right)_{2}^{P}$. Moreover, by Remark 5.1, $\mathfrak{L}_{2}^{P}$ is $\mathbb{K}$-definable. So, since $\mathfrak{L}_{2}^{P}$ is zero-dimensional and $\alpha \notin \mathbb{K}$, one has that $(\alpha, \beta) \notin \mathfrak{L}_{2}^{P}$. Thus, $(\alpha, \beta) \in \mathfrak{Q}_{2} \cup\left(\mathfrak{F}_{\mathcal{P}}\right)_{2}^{P} \subset \pi_{2}\left(\mathfrak{F}_{\mathcal{P}}\right)$. Therefore, there exists $P \in \mathfrak{F}_{\mathcal{P}}$ such that $\pi_{1}(P)=\alpha$. 


\section{References}

[1] Adkins W. A., Wang H.H., Hoffman J.W.: Equations of parametric surfaces with base points via syzygies. Journal of Symbolic Computation. 39/1, 73-101 (2005).

[2] Busé L., Cox D., D'Andrea C.: Implicitization of surfaces in $\mathbb{P}^{3}$ in the presence of base points. Journal of Algebra and Applications. 2, 189-214 (2003).

[3] Busé L., D’Andrea C.: Inversion of Parametrized Hypersurfaces by Means of Hypersurfaces. Proc. ISSAC2004, J. Gutierrez (ed.), 65-79 (2004).

[4] Chen F., Shen L., Deng J.: Implicitization and parametrization of quadratic and cubic surfaces by $\mu$-bases. Anhui. Computing. 79, 131-142 (2007).

[5] Cox D., Goldman R., Zhang M.: On the validity of implicitization by moving quadrics for rational surfaces with no base points. J. Symbolic Computation. 29, 419-440 (2000).

[6] Chionh E. W., Goldman R. N.: Using multivariate resultants to find the implicit equation of a rational surface. The Visual Computer. 8, 171-180 (1992).

[7] Chionh E.W., Goldman R.N.: Degree, Multiplicity and Inversion Formulas for Rational Surfaces using u-Resultants. Computer Aided Geometric Design. 9/2, 93-109 (1992).

[8] Cox D., Little J., O'Shea D.: Ideals, Varieties, and Algorithms (2nd ed.). Springer-Verlag, New York (1997).

[9] Harris J.: Algebraic Geometry. A first Course. Springer-Verlag (1995).

[10] Pérez-Díaz S., Schicho J., Sendra J.R.: Properness and Inversion of Rational Parametrizations of Surfaces. Applicable Algebra in Engineering, Communication and Computing. 13, 29-51 (2002).

[11] Pérez-Díaz S., Sendra J.R.: Computation of the Degree of Rational Surface Parametrizations. Journal of Pure and Applied Algebra. 193/1-3, 99-121 (2004).

[12] Pérez-Díaz S., Sendra J.R.: A Univariate Resultant Based Implicitization Algorithm for Surfaces. Journal of Symbolic Computation. 43, 118-139 (2008).

[13] Pérez-Díaz S., Sendra J.R., Villarino C.: A First Approach Towards Normal Parametrizations of Algebraic Surfaces. International Journal of Algebra and Computation. 20/8, 977-990 (2010).

[14] Pérez-Díaz S., Sendra J.R., Villarino C.: Computing the Singularities of Rational Surfaces. Submitted for publication (arXiv/0289395) (2011).

[15] Sendra J.R., Winkler F.: Computation of the degree of a Rational Map. Proc. ISSAC-2001, B. Mourrain (ed.), 317-322 (2001).

[16] Winkler F.: Polynomials Algorithms in Computer Algebra. Springer-Verlag, Wien, New York (1996).

Sonia Pérez-Díaz

Dpto de Física y Matemáticas

Universidad de Alcalá

E-28871 Madrid

Spain

e-mail: sonia.perez@uah.es

J. Rafael Sendra

Dpto de Física y Matemáticas

Universidad de Alcalá

E-28871 Madrid

Spain

e-mail: rafael.sendra@uah.es 